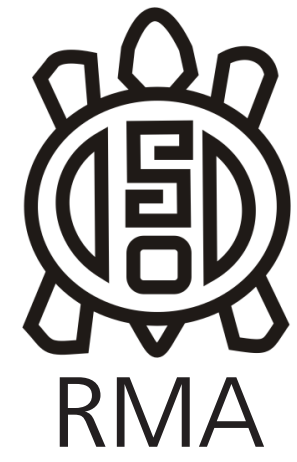

Arqueología

\title{
El rol de la vizcacha (Lagostomus maximus) en los procesos de formación de los sitios arqueológicos de la llanura Interserrana argentina
}

The role of the vizcacha (Lagostomus maximus) in the formation processes of archaeological sites from the Argentinian interhill plains

Jonathan E. Bellinzoni* ${ }^{*}$ Cristian A. Kaufmann** y María Clara Álvarez $z^{* * *}$

*Instituto de Investigaciones Arqueológicas y Paleontológicas del Cuaternario Pampeano (INCUAPA-CONICET), Facultad de Ciencias Sociales, Universidad Nacional del Centro de la Provincia de Buenos Aires, Avda. del Valle 5737, B7400JWI, Olavarría, Buenos Aires, Argentina. E-mail: jonabellinzoni@gmail.com

**Instituto de Investigaciones Arqueológicas y Paleontológicas del Cuaternario Pampeano (INCUAPA-CONICET), Facultad de Ciencias Sociales, Universidad Nacional del Centro de la Provincia de Buenos Aires, Avda. del Valle 5737, B7400JWI, Olavarría, Buenos Aires, Argentina. E-mail: ckaufman@soc.unicen.edu.ar

***Instituto de Investigaciones Arqueológicas y Paleontológicas del Cuaternario Pampeano (INCUAPA-CONICET), Facultad de Ciencias Sociales, Universidad Nacional del Centro de la Provincia de Buenos Aires, Avda. del Valle 5737, B7400JWI, Olavarría, Buenos Aires, Argentina. E-mail: malvarez@soc.unicen.edu.ar

\section{Resumen}

El movimiento de sedimentos que generan los mamíferos cavadores constituye uno de los principales procesos de formación que sufren los sitios arqueológicos de la región pampeana. En este trabajo se presentan los resultados de un estudio actualista realizado sobre una colonia de vizcachas ubicada en la localidad arqueológica El Trebolar, provincia de Buenos Aires. El objetivo principal fue describir el impacto que estos roedores generan sobre el paisaje, con el fin de identificar su accionar en los sitios arqueológicos de la región. A partir del relevamiento de 21 vizcacheras se pudo establecer que las vizcachas afectan al registro arqueológico de diferentes maneras. Por un lado, su actividad cavadora altera las secuencias estratigráficas y genera asociaciones de materiales de distinta cronología. Por otra parte, las vizcacheras son ocupadas por otros animales, dando lugar a conjuntos faunísticos con alta riqueza taxonómica. Además, estos roedores acarrean a sus madrigueras objetos disponibles localmente. Por último, un alto porcentaje de especímenes óseos presentan surcos que generan las vizcachas para mantener funcionales sus incisivos. En conclusión, las distintas evidencias presentadas permitirían reconocer el accionar de este roedor en el registro fósil, diferenciándolo de otros animales fosoriales que habitan la región pampeana.

Palabras claves: Tafonomía actualista; Animales fosoriales; Vizcacheras; Bioturbación; Región pampeana.

\begin{abstract}
The movement of sediments generated by digging animals/burrowing mammals is one of the main formation processes affecting the archaeological sites in the Pampas region. In this article, we present/report the results of an actualistic study conducted on a vizcach a colony, in the archaeological locality El Trebolar, province of Buenos Aires. The main objective was to describe the impact that these rodents generate on the landscape in order to identify their action on the archaeological sites of the region. The analysis of 21 vizcacheras showed that the vizcachas affect the archaeological record in different ways. Firstly, their digging activity alters stratigraphic sequences and generates associations of material of different chronology. Secondly, the vizcacheras are occupied by other animals, producing/giving rise to taxonomically rich faunal assemblages. In addition, these rodents carry locally available objects to their burrows. Finally, a high percentage of bone specimens present/shows grooves generated by vizcachas to keep their incisors functional. In conclusion, evidence would allow recognizing the action of this rodent in the fossil record, distinguishing it from other fossorial animals that inhabit the Pampas region.
\end{abstract}

Keywords: Actualistic taphonomy; Fossorial animals; Vizcacheras; Bioturbation, Pampas region. 


\section{Introducción}

La mayoría de los sitios arqueológicos de la región pampeana argentina se ubican a cielo abierto y se emplazan en suelos de naturaleza dinámica, los cuales están sujetos a diversos procesos biomecánicos (Zárate et al. 2000). Entre estos últimos se destaca la faunaturbación (Waters 1992), definida como la mezcla y el desplazamiento constante de los sedimentos a través de la acción de animales de hábitos cavadores, insectos y anélidos (Wood y Johnson 1978; Johnson 1990, 2002; Balek 2002). En las planicies pampeanas, los estudios acerca de la acción perturbadora de diversos mamíferos tales como armadillos, coipos y cuises, se iniciaron hace varias décadas (e.g., Politis y Madrid 1988; Frontini y Escosteguy 2012; Álvarez et al. 2020). Recientemente, se ha comenzado a evaluar de forma sistemática el rol de las vizcachas (Lagostomus maximus) en la perturbación de la estratigrafía y el registro fósil (Rafuse et al. 2019; Tomassini et al. 2019).

La vizcacha presenta diversas características que influyen en la forma en que este agente puede afectar a los depósitos arqueológicos. En primer lugar, puede mover materiales sepultados hacia la superficie durante la construcción de sus cuevas (Rafuse et al. 2019; Tomassini et al. 2019). Además, este roedor acarrea al entorno de sus madrigueras diversos objetos que se encuentran en su rango de forrajeo (Villarreal et al. 2008; Rafuse et al. 2019). Otro aspecto importante es que estos roedores constituyen verdaderos ingenieros ecosistémicos (Arias et al. 2005). En este sentido, la construcción de sus cuevas genera un impacto sobre la vegetación y la denudación e infiltración del agua en los suelos (Branch et al. 1996; Campos 1997; Arias et al. 2003, 2005). Además, las vizcachas pueden modificar la composición química de los suelos, aumentando los niveles de fósforo como consecuencia de la movilización y acumulación de toscas (Kelt 2011; Platt et al. 2016). Finalmente, este chinchíllido, al igual que otros roedores, puede generar marcas sobre diversos restos materiales (e.g., huesos), a partir de su roído (Jackson et al. 1996; Giuletti y Veneciano 2005).

En lo que respecta a la explotación de la vizcacha por parte de las poblaciones humanas, el aprovechamiento de su carne y cuero tiene una larga tradición histórica en distintas regiones de la Argentina (Mares y Ojeda 1984; Jackson 1986; Jackson et al. 1996). Asimismo, su consumo se registra en sitios arqueológicos de la región pampeana con cronologías que abarcan todo el Holoceno (Martínez et al. 2001; Mazzanti y Quintana 2001; Martínez y Gutiérrez 2004; Salemme y Madrid 2007; Bonomo et al. 2008; Politis y Messineo 2008; Álvarez 2009, 2014; Quintana y Mazzanti 2011; Escosteguy y Salemme 2012; Álvarez et al. 2013; Messineo et al. 2013; Massigoge et al. 2018). Sin embargo, el origen de los huesos de vizcacha en los conjuntos faunísticos puede tener un origen complejo. Estos roedores, de manera coincidente con los grupos humanos, suelen ocupar lugares altos y drenados del paisaje, cercanos a fuentes de agua (Politis y Madrid 1988). Esta superposición espacial aumenta las chances de que los restos óseos de vizcacha presentes en los sitios arqueológicos tengan un origen mixto (Escosteguy y Salemme 2012; Salemme et al. 2012). Es decir, algunos de sus restos podrían haberse depositado como producto del aprovechamiento humano y otros como consecuencia de la depredación por carnívoros y aves rapaces o por muertes eto-ecológicas (sensu Pardiñas 1999:269).

Durante los últimos diez años, hemos llevado a cabo investigaciones arqueológicas sistemáticas en la microrregión de la Cuenca del arroyo Salado, ubicada en el área Interserrana bonaerense (Kaufmann y González 2013; Barros et al. 2018a, 2018b; Kaufmann et al. 2019; Álvarez et al. 2020; Barros et al. 2020). Los restos de vizcacha han sido registrados en los depósitos arqueológicos estratigráficos y superficiales de esta microrregión, así como en vizcacheras activas y abandonadas identificadas en sus alrededores. En el sitio Hangar, con cronologías del Holoceno tardío final, se recuperaron restos de vizcacha con huellas de corte asignadas a cuereo (Barros et al. 2018a). Por otra parte, en el sitio Laguna Muscar 2, que tiene un fechado del Holoceno medio, se recuperaron especímenes de este roedor, aunque se propuso que su origen habría sido natural y posterior a las ocupaciones humanas (Bellinzoni 2018). En el sector 2 de este sitio se relevó un entierro humano primario con fechados del Holoceno tardío, cuyas unidades anatómicas fueron desplazadas por la acción de las vizcachas, que mezclaron sedimentos y horizontes de suelo (Kaufmann et al. 2019). Las observaciones mencionadas acerca de este chinchíllido nos llevaron a realizar, en el año 2014, un estudio actualista con el objetivo principal de describir el impacto potencial de las vizcachas sobre los sitios arqueológicos del área. En este trabajo se presentan los resultados de dicha investigación, que fue realizada sobre un vizcacheral que se encontraba activo al momento de las observaciones. Este se localiza en los bordes de una pequeña laguna, en la localidad arqueológica El Trebolar, en el partido de General Lamadrid, provincia Buenos Aires, Argentina (Figura 1).

\section{La vizcacha de la llanura}

La vizcacha o vizcacha de la llanura es un roedor que habita matorrales y pastizales de tierras bajas desde el sur de Bolivia hasta el norte de la Patagonia argentina (Figura 1) (Cabrera 1961). Este animal es la especie más grande y de mayor dimorfismo sexual de los chinchíllidos, llegando los machos adultos a los $9 \mathrm{~kg}$ y las hembras a los 4,5 kg (Weir 1974). La vizcacha consume una amplia variedad de hierbas y pequeños arbustos cercanos a las vizcacheras, aunque puede alejarse hasta $400 \mathrm{~m}$ cuando el alimento es escaso 


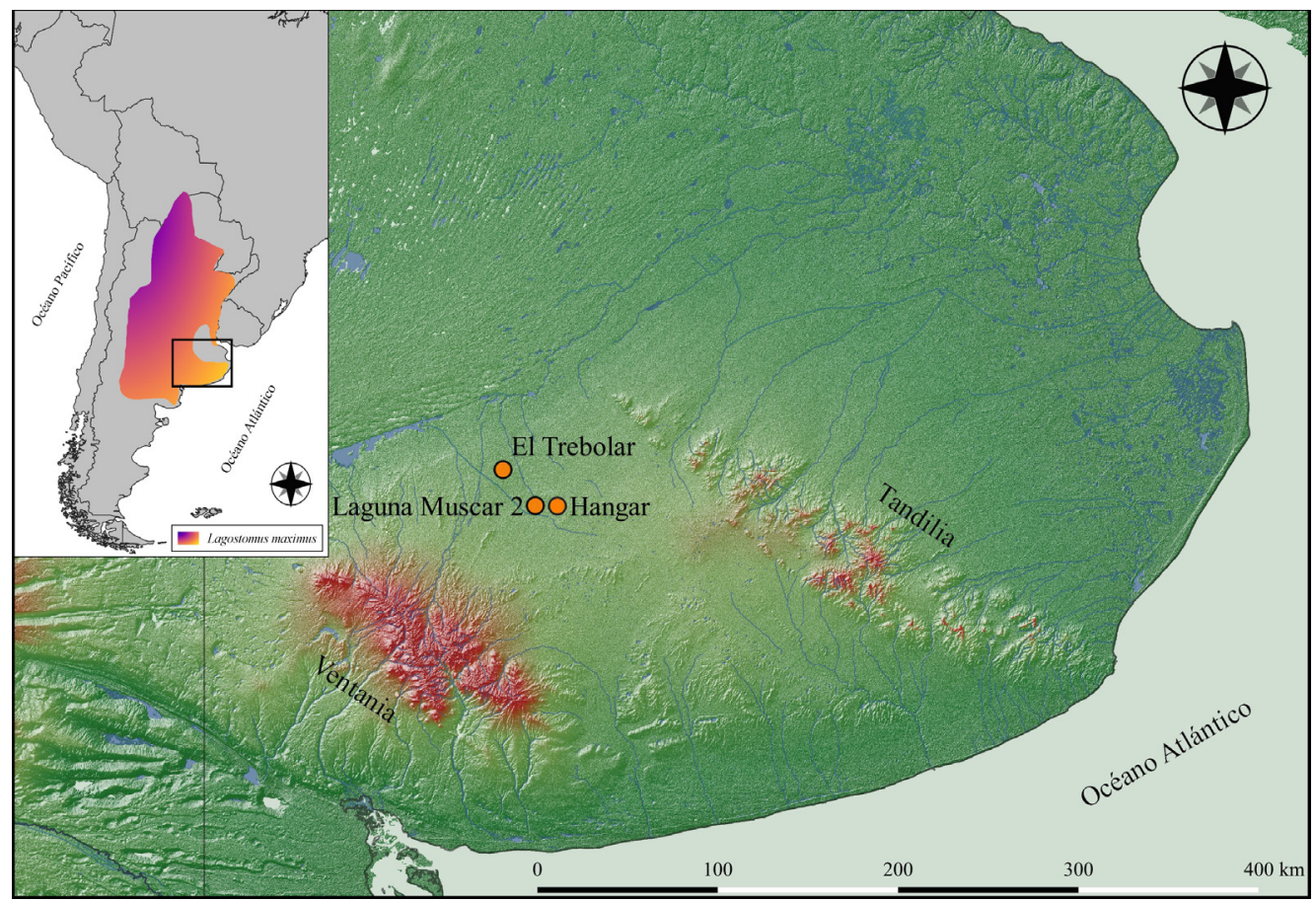

Figura 1. Distribución actual de vizcacha y ubicación de las localidades arqueológicas del área de estudio.

Figure 1. Actual distribution of vizcacha and location of the archaeological localities from the study area.

(Jackson 1986; Branch y Sosa 1994; Jofré 1994). La reproducción de este roedor es estacional; la mayoría de las hembras entran en celo en otoño y los nacimientos suceden hacia la primavera, dando lugar a una o dos crías (Llanos y Crespo 1952). Las vizcachas son animales sociales que viven en grupos de 10 a 30 individuos, integrados por 1 a 3 machos y varias hembras adultas con sus crías. Cada grupo social ocupa un sistema comunal de madrigueras, conocido como vizcachera, la cual puede ser utilizada por varias generaciones (Llanos y Crespo 1952).

Los sistemas de madrigueras suelen presentar entre 10 y 100 cuevas que están interconectadas por túneles que desembocan en cámaras subterráneas que pueden alcanzar los $2 \mathrm{~m}$ de diámetro y $2 \mathrm{~m}$ de profundidad (Jackson et al. 1996). Cada grupo establece pequeños sistemas de madrigueras satélites, que suelen presentar entre 1 y 10 entradas. Estas vizcacheras auxiliares generalmente son utilizadas como refugio temporal de los machos adultos (Branch et al. 1993). Una característica particular de la vizcacha es que acarrea diversos objetos a sus madrigueras, que son acumulados alrededor de las entradas de las cuevas (Villareal et al. 2008).

Algunos de los depredadores nativos de la vizcacha son el zorro pampeano (Lycalopex gymnocercus), el hurón (Galictis cuja), el gato montés (Leopardus geoffroyi) y la boa de las vizcacheras (Boa constrictor) (Jackson et al. 1996). Actualmente, este chinchíllido ha sido categorizado como de Preocupación Menor (LC) para el riesgo de extinción en la lista roja de los mamíferos de Argentina. No obstante, varias poblaciones del Chaco Seco, Pampa y Espinal persisten en bajas densidades
(Cirignoli y Lartigau 2019)

\section{Materiales y métodos}

\section{Localidad arqueológica El Trebolar}

El Trebolar (37 $03^{\prime} \mathrm{S} ; 61^{\circ} 27^{\prime} \mathrm{O}$ ) es una localidad arqueológica ubicada en una pequeña laguna que se encuentra atravesada por un curso de agua, tributario del arroyo Salado. Este curso fue canalizado por obras hidráulicas modernas, lo cual redujo notablemente el volumen de agua de la laguna. La prospección realizada sobre las márgenes permitió identificar un sitio con desechos líticos depositados superficialmente sobre un sector desprovisto de vegetación. Durante las tareas de campo también se observó la presencia de numerosas vizcacheras activas sobre los bordes de la laguna, aspecto que motivó la realización del presente estudio actualista.

\section{Relevamiento de las vizcacheras en el campo}

La identificación y registro de las vizcacheras se llevó a cabo a partir de la recorrida a pie de las márgenes de la laguna, tarea que comprendió el relevamiento de ca. $250.000 \mathrm{~m}^{2}$. Cada una de las vizcacheras y sus bocas de entrada fueron georreferenciadas. Sin embargo, no se realizó una distinción entre vizcacheras principales y auxiliares o satélites. Se contabilizó el número de bocas presentes y se registraron los diferentes materiales asociados al contexto de las madrigueras. A partir de la información contextual y características de los objetos se definieron cuatro grupos de ítems asociados: G1) Huesos modernos y heces de carnívoros depositadas naturalmente en el contexto de las madrigueras (e.g., carcasas de animales depositadas por depredadores o por muertes eto-ecológicas); G2) Huesos modernos de 
vizcachas que corresponden a individuos que habrían muerto dentro de la vizcachera y fueron transportados a la superficie durante las actividades de mantenimiento y limpieza de las cuevas (ver Tomassini et al. 2019); G3) Objetos exhumados durante la excavación de las cuevas (e.g., huesos y toscas que forman parte de la matriz sedimentaria y se encontraban asociados al sedimento removido) y G4) Objetos acarreados por las vizcachas al entorno de sus madrigueras (e.g., tallos y ramas, bosta de vaca seca, huesos, plásticos, gomas, etc.).

Se recolectaron todos los restos óseos asociados con las vizcacheras $\mathrm{N}^{\circ} 2,4,7,8$ y 11 para su análisis taxonómico y tafonómico en el laboratorio. En las vizcacheras $N^{\circ} 7,8$ y 11 se realizó un relevamiento planimétrico georreferenciado de las cuevas y los materiales asociados. Los datos fueron procesados con el software libre SIG QGIS (versión 3.8.3). Una de las vizcacheras ( $\left.N^{\circ} 11\right)$ se encontraba inactiva; motivo por el cual fue excavada con el fin de explorar su estructura. La excavación se realizó siguiendo la distribución de las madrigueras. Además, en la mitad de la vizcachera se realizó una trinchera de $3 \mathrm{~m}$ de largo x 0,5 $\mathrm{m}$ de ancho y $1 \mathrm{~m}$ de profundidad.

Los conjuntos $\mathrm{N}^{\circ} 7$ y 8 se encontraban asociados con una carcasa moderna de vaca. Algunos de sus huesos fueron transportados por los roedores hasta las vizcacheras. Con el fin de vincular y establecer las distancias a la que fueron transportados los restos óseos, se llevaron a cabo remontajes de tipo mecánico y anatómico (Todd 1987; Todd y Frison 1992; Todd y Stanford 1992). Los remontajes mecánicos se realizaron acoplando dos o más fragmentos pertenecientes a un mismo elemento óseo a partir de la superficie de fractura. Por otra parte, los remontajes anatómicos consistieron en la recomposición de las relaciones intraesqueletarias entre los elementos óseos. Estas se reconocieron considerando las correspondencias bilaterales e intermembrales. Las primeras refieren a la unión entre elementos anatómicos pares (e.g., húmero derecho y húmero izquierdo) y las segundas entre elementos que articulan entre sí (e.g., fémur derecho y tibia derecha).

\section{Estudio faunístico y tafonómico de laboratorio}

En primera instancia, se llevó a cabo la identificación taxonómica y anatómica de los elementos óseos del conjunto superficial depositado en el contexto de las vizcacheras. Para esto se utilizaron las colecciones de referencia de la UE-INCUAPA-CONICET. En relación con las medidas de abundancia taxonómica, se calculó el número mínimo de especímenes (NISP) y el número mínimo de individuos (MNI). Asimismo, se estimó la riqueza taxonómica de la muestra a través del NTAXA. Para el caso específico de los restos de vizcacha se calculó también el número mínimo de elementos (MNE) y la abundancia relativa (Ri) (Andrews 1990; Lyman 1994, 2008).

El accionar de distintos agentes tafonómicos sobre el conjunto fue evaluado a partir del relevamiento de diferentes variables que fueron observadas con lupa binocular de 20x y 40x. Los efectos considerados fueron meteorización (Behrensmeyer 1978), vermiculaciones (Fisher 1995), osteofagia (Cáceres et al. 2013) y daños generados por carnívoros (perforaciones, hoyuelos y ahuecado) (Haynes 1980; Binford 1981). En este registro también fueron consideradas las modificaciones de origen antrópico como huellas de corte, raspado y percusión (Mengoni Goñalons 1999). Teniendo en cuenta que los roedores pueden generar una gran diversidad de marcas (Politis y Madrid 1988), se describieron en detalle los surcos presentes en los huesos. Para este registro se tuvieron en cuenta las principales variables que son consideradas por los investigadores que han abordado esta problemática (Brain 1981; Politis y Madrid 1988; Quintana 2007). Estas son largo, ancho, profundidad, sección, frecuencia, localización topográfica y orientación

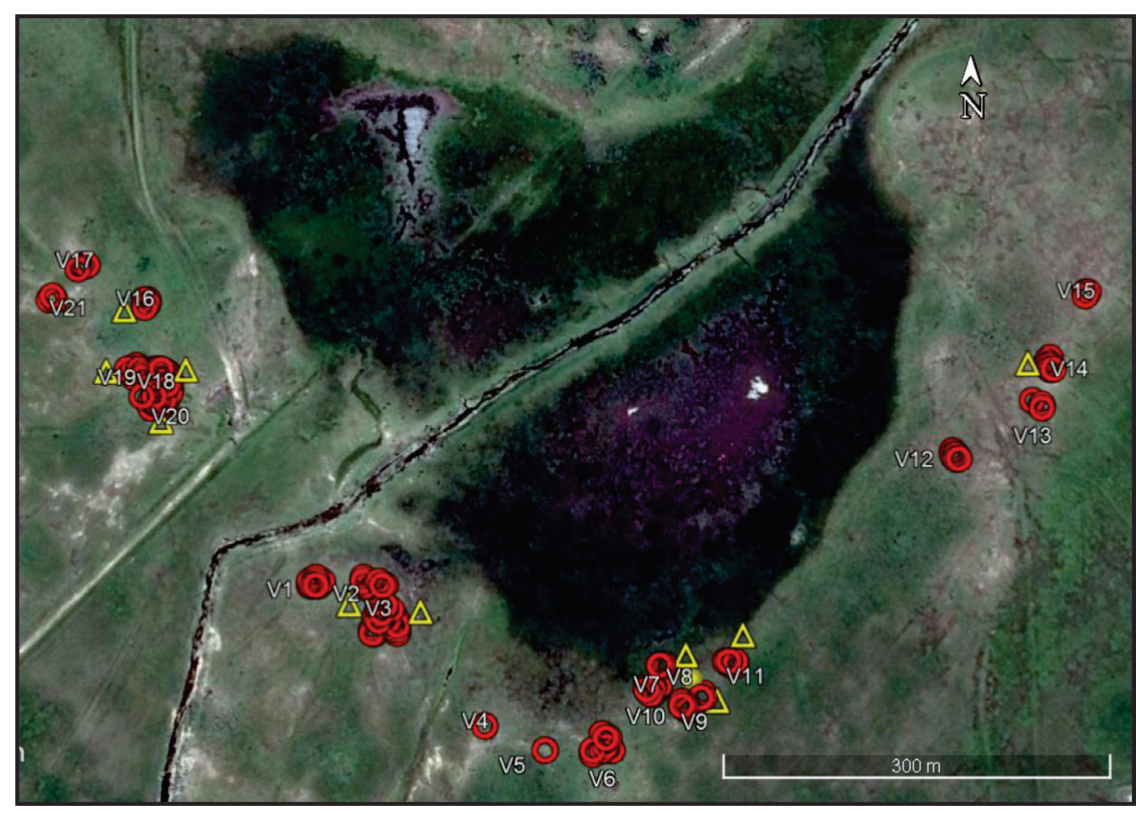

Figura 2. Localidad arqueológica El Trebolar con detalle de la distribución de las vizcacheras relevadas en este trabajo.

Figure 2. Archaeological locality El Trebolar and detail of the vizcacheras distribution recorded in this work. 


\begin{tabular}{|c|c|c|c|c|c|c|c|c|c|c|c|c|c|c|c|c|c|c|}
\hline \multirow{2}{*}{ Viz. } & \multirow{2}{*}{ NB } & \multirow{2}{*}{$M^{2}$} & \multicolumn{4}{|c|}{ G1 } & \multirow{2}{*}{\begin{tabular}{|l|} 
G2 \\
5
\end{tabular}} & \multicolumn{4}{|c|}{ G3 } & \multicolumn{7}{|l|}{ G4 } \\
\hline & & & 1 & 2 & 3 & 4 & & 6 & 7 & 8 & 9 & 10 & 11 & 12 & 13 & 14 & 15 & 16 \\
\hline$N^{0} 1$ & 16 & 48 & & & & & & & & & & & & & & & & \\
\hline$N^{\circ} 2$ & 29 & 94,4 & & & & & & & & & & & & & & & & \\
\hline$N^{\circ} 3$ & 30 & 175 & & & & & & & & & & & & & & & & \\
\hline$N^{\circ} 4$ & 13 & 102 & & & & & & & & & & & & & & & & \\
\hline$N^{\circ} 5$ & 9 & 94,5 & & & & & & & & & & & & & & & & \\
\hline$N^{\circ} 6$ & 8 & 169 & & & & & & & & & & & & & & & & \\
\hline$N^{\circ} 7$ & 9 & 44,2 & & & & & & & & & & & & & & & & \\
\hline$N^{\circ} 8$ & 8 & 5 & & & & & & & & & & & & & & & & \\
\hline$N^{\circ} 9$ & 5 & 4,64 & & & & & & & & & & & & & & & & \\
\hline$N^{\circ} 10$ & 10 & 19 & & & & & & & & & & & & & & & & \\
\hline$N^{\circ} 11$ & 8 & 18,4 & & & & & & & & & & & & & & & & \\
\hline$N^{\circ} 12$ & 6 & 15,3 & & & & & & & & & & & & & & & & \\
\hline$N^{\circ} 13$ & 6 & 14,3 & & & & & & & & & & & & & & & & \\
\hline$N^{\circ} 14$ & 8 & 28,8 & & & & & & & & & & & & & & & & \\
\hline$N^{\circ} 15$ & 6 & 10,6 & & & & & & & & & & & & & & & & \\
\hline$N^{\circ} 16$ & 6 & 17,4 & & & & & & & & & & & & & & & & \\
\hline$N^{\circ} 17$ & 4 & 19,5 & & & & & & & & & & & & & & & & \\
\hline$N^{\circ} 18$ & 10 & 95,4 & & & & & & & & & & & & & & & & \\
\hline$N^{\circ} 19$ & 17 & 119 & & & & & & & & & & & & & & & & \\
\hline$N^{\circ} 20$ & 13 & 122 & & & & & & & & & & & & & & & & \\
\hline \multirow[t]{2}{*}{$N^{0} 21$} & 3 & 9,85 & & & & & & & & & & & & & & & & \\
\hline & 224 & 1226,3 & 5 & 2 & 1 & 5 & 15 & 2 & 2 & 10 & 11 & 10 & 11 & 9 & 4 & 2 & 3 & 1 \\
\hline
\end{tabular}

Tabla 1. Número de bocas, $\mathrm{m}^{2}$ y materiales hallados en las vizcacheras.

Table 1. Number of entrances, $m^{2}$, and materials found in the vizcacheras.

Referencias: Viz.=vizcachera; NB=número de bocas; G1:1=armadillos; $2=L$. gymnocercus; $3=$ Aves; $4=$ Heces de carnívoro. G2: $5=$ L. maximus. G3: $6=$ L. guanicoe; 7=R. americana; $8=$ lítico; 9=toscas. G4: $10=B$. Taurus; $11=$ tallos secos; $12=$ bosta seca; 13=metal; 14=goma; 15=plástico; $16=$ vidrio.

de los surcos sobre los huesos.

\section{Resultados}

\section{Características de las vizcacheras relevadas}

La prospección realizada en los bordes de la laguna permitió registrar 21 vizcacheras compuestas por un total de 224 bocas de entrada (Figura 2). El número mínimo de bocas de entrada por vizcachera fue de 3, el máximo de 30 y el promedio de 10,5. El promedio del área que ocupaba cada vizcachera fue de $58,4 \mathrm{~m}^{2}$, con un mínimo de 4,6 $\mathrm{m}^{2}$ y un máximo de $175 \mathrm{~m}^{2}$. El total del área afectada directamente por las madrigueras fue de 1226,3 $\mathrm{m}^{2}$.

La excavación de la vizcachera N11 permitió observar que la mayoría de los túneles desembocaban directamente en la cámara central, mientras que un número menor se conectaba con otras galerías y corredores. Las dimensiones de los túneles variaron entre 20 y $35 \mathrm{~cm}$ de ancho y 15 y $20 \mathrm{~cm}$ de alto, mientras que la cámara central presentaba $75 \mathrm{~cm}$ de largo, $50 \mathrm{~cm}$ de ancho y $35 \mathrm{~cm}$ de alto. La profundidad de la cámara central en la que desembocaban los túneles alcanzó los $60 \mathrm{~cm}$ de profundidad.

Del total de vizcacheras analizadas, en 19 (90,5\%) se registró algún tipo de material asociado (Tabla 1). El $52,4 \%(N=11)$ de las vizcacheras presentaba restos óseos modernos (G1) (Tabla 1). Los ítems más frecuentes dentro de esta categoría fueron las heces de carnívoros y los restos de armadillos (23,8\%), seguidos por huesos de zorro pampeano (Lycalopex gymnocercus) (9,5\%) y aves $(4,8 \%)$. En el $71,4 \%(\mathrm{~N}=15)$ de las madrigueras se relevaron restos óseos de vizcacha (G2) (Tabla 1). Por otra parte, el $76,2 \%(\mathrm{~N}=16)$ de las vizcacheras presentaba materiales asociados con los conos de sedimento generados durante la excavación de las madrigueras (G3) (Tabla 1). Los restos más frecuentes en este grupo fueron las toscas de carbonato de calcio (presentes en el 52,4\% de las vizcacheras), que forman parte de la secuencia estratigráfica de la laguna. En orden de importancia siguen los artefactos líticos (presentes en el $47,6 \%$ de las vizcacheras), los huesos de ñandú (presentes en el 9,5\% de las vizcacheras) y los de guanaco (presentes en el 9,5\% de las vizcacheras). En $18(85,7 \%)$ de las vizcacheras se identificaron objetos acarreados por los roedores al entorno de sus madrigueras (G4) (Tabla 1). Los más frecuentes fueron los tallos secos de plantas (posiblemente cardos) y las toscas de carbonato de calcio (52,4\% en ambos casos), los huesos de vaca $(47,6 \%)$ y la bosta seca $(42,8 \%)$. De forma ocasional se relevaron metales (latas, alambres y varillas) (19\%), plásticos $(14,3 \%)$, goma $(9,5 \%)$ y vidrio (botella) $(4,8 \%)$ (Figura 3).

\section{Análisis espacial de los restos óseos de las vizcacheras $\mathrm{N}^{\circ} 7$ y 8}

Se llevó a cabo el análisis detallado de la distribución espacial de las cuevas y de los materiales hallados en la superficie de las vizcacheras $N^{\circ} 7$ y 8 . En ambas se identificaron huesos correspondientes a una carcasa de vaca (Bos taurus) que estaba depositada en un sector cercano. Este hallazgo resultó muy informativo 


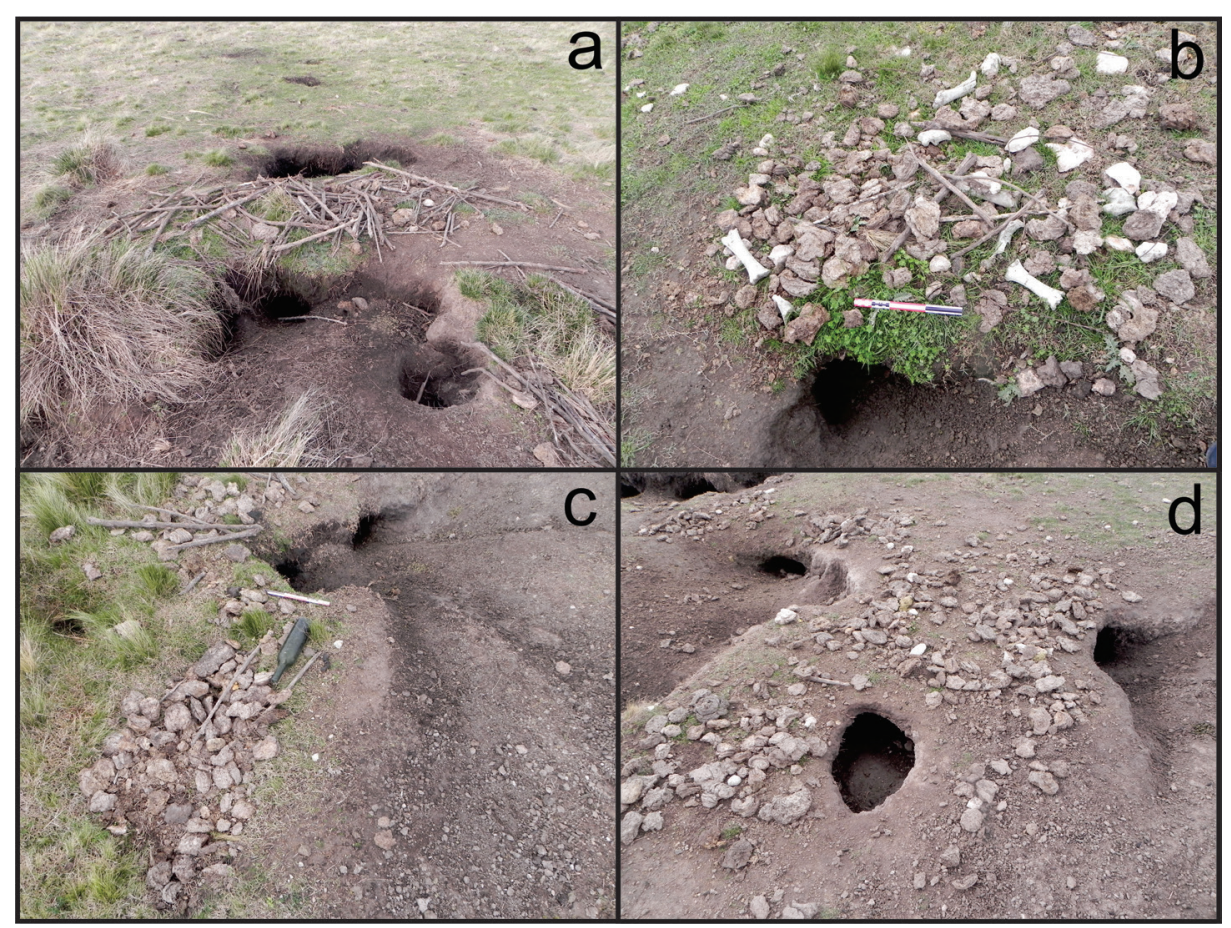

Figura 3. Vizcacheras con diversos objetos depositados en su entorno. A) Vizcachera $\mathrm{N}^{\circ} 16$ con abundantes tallos secos y bosta de vaca depositada sobre las madrigueras; B) Vizcachera $\mathrm{N}^{\circ} 13$ con depósito de huesos de vaca, tallos secos y bosta de vaca; C) Vizcachera $\mathrm{N}^{\circ} 6$ en la cual se puede observar una botella de vidrio, una hez de carnívoro y abundantes tallos secos y bosta de vaca. El sedimento de la entrada de la madriguera se encuentra recientemente removido por los roedores y; D) Vizcachera $\mathrm{N}^{\circ}$ 15 en la cual se observa tallos secos, bosta de vaca y colapso del techo de algunas madrigueras.

Figure 3. Vizcacheras with several objects deposited in their surroundings: A) Vizcachera $\mathrm{N}^{\circ} 16$ with abundant dry stems and dung cow deposited on the burrows; B) Vizcachera $\mathrm{N}^{\circ} 13$ with cow bones, dry stems, and cow dung; C) Vizcachera $\mathrm{N}^{\circ}$ 6 with glass bottle, carnivore feces, and abundant dry stems and cow dung. The sediment in the burrow entrance has been recently removed by rodents, and D) Vizcachera $\mathrm{N}^{\circ} 15$ with dry stems, cow dung, and collapsed roof in some of the burrows. en relación con las distancias y pesos de los objetos que las vizcachas pueden transportar a sus madrigueras.

El conjunto $\mathrm{N}^{\circ} 7$ está emplazado sobre una pequeña lomada próxima al cuerpo de agua. Se trata de una vizcachera activa compuesta por 10 entradas. El área comprende $44 \mathrm{~m}^{2}$ y se encuentra modificada en su totalidad por las madrigueras y el sedimento removido. La vegetación se desarrolla exclusivamente en el centro de la vizcachera, donde se observan diversos objetos como bosta seca, tosca, metal, plástico y restos óseos de vaca $(\mathrm{NISP}=18)$. Estos últimos corresponden a costillas $(N=10)$, vértebras cervicales $(N=3)$, vértebras torácicas $(\mathrm{N}=2)$, escápula $(\mathrm{N}=1)$, cúbito $(\mathrm{N}=1)$ y hemipelvis $(\mathrm{N}=1)$.

La vizcachera $\mathrm{N}^{\circ} 8$ está integrada por cuatro madrigueras (una de ellas colapsada) y se ubica sobre la línea de

Tabla 2. Correspondencias anatómicas de vaca realizadas en las vizcacheras $\mathrm{N}^{\circ} 7,8$ y carcasa asociada.

Table 2. Anatomical correspondences of cow in vizcacheras $\mathrm{N}^{\circ} 7,8$, and associated carcass.

$\mathrm{UAV}=$ unidades de análisis vinculadas; $\mathrm{UB}=$ uniones bilaterales; $\mathrm{UI}=$ uniones intermembrales; $\mathrm{UM}=$ uniones mecánicas.

\begin{tabular}{|c|c|c|c|}
\hline Correspondencia & Elementos óseos vinculados & UAV & Distancia \\
\hline UB & Fémur izq. y der. & Fy V8 & $21,9 \mathrm{~m}$ \\
\hline UB & Calcáneo izq. y der. & Fy V7 & $33,6 \mathrm{~m}$ \\
\hline UB & Costilla izq. y der. & V7 y V8 & $22,3 \mathrm{~m}$ \\
\hline UB & Hemipelvis izq. y der. & V7 y V8 & $19,4 \mathrm{~m}$ \\
\hline $\mathrm{UI}$ & V. axis y atlas & Fy V7 & $32,7 \mathrm{~m}$ \\
\hline $\mathrm{UI}$ & Fémur izq. y hemipelvis izq. & Fy V7 & $30,9 \mathrm{~m}$ \\
\hline $\mathrm{UI}$ & Húmero y cúbito der. & F y V7 & $35,9 \mathrm{~m}$ \\
\hline $\mathrm{UI}$ & Fémur y tibia der. & Fy V8 & $24,0 \mathrm{~m}$ \\
\hline UI & Húmero y radio der. & Fy V8 & $20,0 \mathrm{~m}$ \\
\hline $\mathrm{UI}$ & Hemimandíbula der. y Cráneo & Fy V8 & $22,2 \mathrm{~m}$ \\
\hline $\mathrm{UI}$ & Hemimandíbula izq. y Cráneo & Fy V8 & $20,9 \mathrm{~m}$ \\
\hline $\mathrm{UI}$ & Cráneo y V. atlas & V7 y V8 & $22,2 \mathrm{~m}$ \\
\hline UI & Sacro y hemipelvis izq. & V7 y V8 & $21,4 \mathrm{~m}$ \\
\hline UM & Radiocúbito ds. der. y cúbito der. & $\vee 7$ y $\vee 8$ & $23,6 \mathrm{~m}$ \\
\hline UM & Radio der. y cúb. der & $\vee 7$ y $\vee 8$ & $22,8 \mathrm{~m}$ \\
\hline UM & Fémur D. y Fémur ds. & Fy $\vee 8$ & $22,3 \mathrm{~m}$ \\
\hline
\end{tabular}




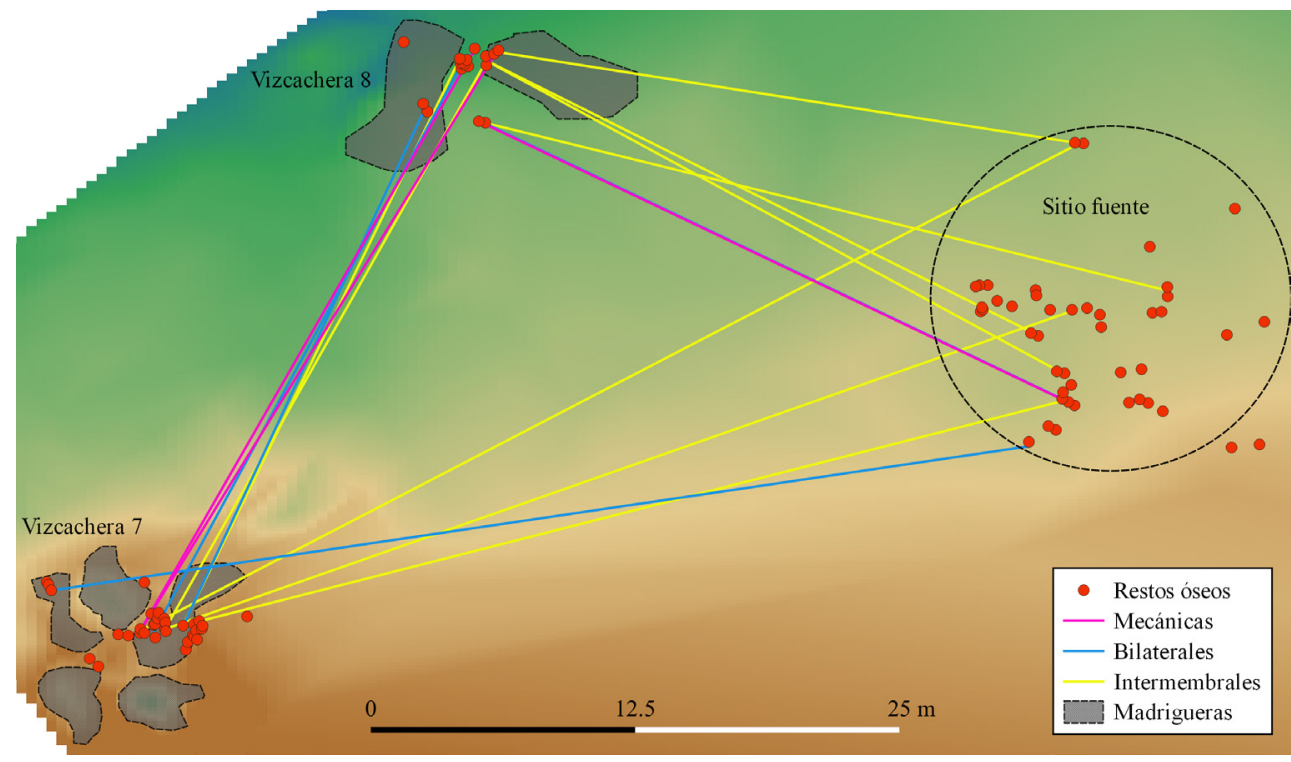

Figura 4. Mapa planimétrico de las vizcacheras $\mathrm{N}^{\circ}$ 7 y 8 con detalle de las correspondencias anatómicas realizadas.

Figure 4. Planimetric map of vizcacheras $\mathrm{N}^{\circ} 7$ and 8 with detail of the anatomical correspondences.

costa de la laguna. Este conjunto, que al momento de la observación se encontraba inactivo por la inundación de sus madrigueras, podría corresponder a una estructura satelital de la vizcachera $\mathrm{N}^{\circ} 7$ (dista $27 \mathrm{~m}$ de esta). El área en la cual se extiende la vizcachera $\left(5 \mathrm{~m}^{2}\right)$ se encuentra vegetada homogéneamente y no se registra presencia de conos de sedimento, ni parches de vegetación. El material asociado incluye dos desechos líticos y restos óseos de vaca (NISP=12). Estos restos corresponden a cráneo $(\mathrm{N}=1)$, vertebra torácica $(\mathrm{N}=1)$, vértebra lumbar $(\mathrm{N}=2)$, sacro $(\mathrm{N}=1)$, extremidad proximal del sacro $(\mathrm{N}=1)$, hemipelvis $(\mathrm{N}=1)$, costilla $(\mathrm{N}=2)$, fémur $(\mathrm{N}=1)$, radio $(\mathrm{N}=1)$ y cúbito $(\mathrm{N}=1)$.

En un sector cercano, a $31 \mathrm{~m}$ del de la vizcachera $\mathrm{N}^{\circ} 7$ y a 23,7 m de la vizcachera $N^{\circ} 8$, se reconoció la fuente desde la cual las vizcachas transportaron los huesos. Al momento de la observación se hallaron varios huesos de vaca (NISP=33), algunos de ellos aún articulados. Los restos corresponden a ambas hemimandíbulas $(\mathrm{N}=2)$, vértebras cervicales $(\mathrm{N}=3)$, vértebras torácicas $(\mathrm{N}=8)$, costillas $(\mathrm{N}=8)$, diáfisis de fémur $(\mathrm{N}=1)$, epífisis distal de fémur $(\mathrm{N}=1)$, tibias $(\mathrm{N}=2)$, calcáneos $(\mathrm{N}=2)$, escápula $(\mathrm{N}=1)$, húmero $(\mathrm{N}=1)$, metatarso $(\mathrm{N}=1)$, cuneiforme $(\mathrm{N}=1)$, navículo-cuneiforme $(\mathrm{N}=1)$ y primera falange $(\mathrm{N}=1)$. El grado de desarrollo de los dientes (Getty et al. 1982) y el estado de fusión de los huesos (Barone 1999) permitió determinar al individuo como subadulto (30 a 36 meses).

Se realizaron distintas correspondencias anatómicas entre los huesos, que comprenden cuatro uniones bilaterales, nueve intermembrales y tres mecánicas (Figura 4, Tabla 2). Estas correspondencias involucraron un promedio de dispersión de los huesos de 24,8 m, con un mínimo de $20 \mathrm{~m}$ y un máximo de $35,9 \mathrm{~m}$.

Una vez que se estableció que los huesos de las tres unidades de análisis descriptas correspondían a un único individuo, se procedió a estimar la distancia existente entre 28 especímenes óseos depositados en las vizcacheras $\mathrm{N}^{\circ}$ 7 y 8 con el sitio fuente. Asimismo, se registró el peso de 31 especímenes óseos y 62 toscas depositadas sobre la vizcachera $\mathrm{N}^{\circ} 7$. El promedio de distancia de los huesos fue de 33,1 m, con un mínimo de 24,6 m y un máximo de 40,3 m. Por otra parte, el peso promedio de estos fue de 169, 1 gr, con un mínimo de 31 gr (costilla) y un máximo de 1583 gr (cráneo). Finalmente, el peso promedio de las toscas fue de 417,2 gr, con un mínimo de $10 \mathrm{gr}$ y un máximo de $1560 \mathrm{gr}$.

\section{Análisis taxonómico de los restos óseos}

A través del relevamiento superficial de cinco vizcacheras se recolectó un total de 262 especímenes óseos. Un $73,3 \%$ pudo ser asignado a algún nivel taxonómico, lo cual derivó en un NTAXA de nueve, en el que dominan ampliamente los mamíferos (Tabla 3). El taxón mejor representado es la vaca $(26,7 \%)$, seguido por la vizcacha $(15,3 \%)$ y el zorro pampeano $(13,7 \%)$. El resto de los taxones como guanaco, Paseriformes, Strigiformes, ñandú, mulita (Dasypus hybridus) y Anura, están representados por muy pocos especímenes. El $38,5 \%$ de la muestra corresponde a fragmentos óseos indeterminados menores a $4 \mathrm{~cm}$.

La recurrencia de huesos de vizcacha en el entorno de las cuevas se relacionaría con muertes eto-ecológicas dentro de las madrigueras o depredación. Los huesos asignados a zorro pampeano corresponden a una carcasa moderna que aún presentaba tejido blando. Los huesos de Paseriformes, Anura y Aves provienen de la única hez de carnívoro en la muestra. Los restos de ñandú, mulita y guanaco presentan tinción por enterramiento y, al igual que las toscas, habrían sido exhumados por la actividad cavadora de las vizcachas. De estos taxones, el único que evidencia un origen antrópico es el guanaco. Los siete 


\begin{tabular}{|c|c|c|c|c|}
\hline Clase & Orden & Especie & NISP & NISP\% \\
\hline Mammalia & Artiodactyla & Bos taurus & 70 & 26,7 \\
\hline Mammalia & Artiodactyla & Lama guanicoe & 7 & 2,7 \\
\hline Aves & Struthioniformes & Rhea americana & 1 & 0,4 \\
\hline Mammalia & Carnivora & Lycalopex gymnocercus & 36 & 13,7 \\
\hline Mammalia & Rodentia & Lagostomus maximus & 40 & 15,3 \\
\hline Mammalia & Cingulata & Dasypus hybridus & 1 & 0,4 \\
\hline $\begin{array}{c}\text { Aves } \\
\text { pequeña) }\end{array}$ & & & 1 & 0,4 \\
\hline Aves & Strigiformes & & 1 & 0,4 \\
\hline Aves & Passeriformes & 3 & 1,1 \\
\hline Amphibia & Anura & 1 & 0,4 \\
\hline \multicolumn{2}{|r|}{ Indeterminado } & 101 & 38,5 \\
\hline \multicolumn{2}{|c|}{ Total } & 262 & 100 \\
\hline
\end{tabular}

Tabla 3. Representación taxonómica de la muestra ósea.

Table 3. Taxonomic representation of the bone sample. restos de este taxón provienen del cono de sedimento de la vizcachera $\mathrm{N}^{\circ} 11$ y se encontraban asociados con 11 artefactos líticos (ocho de cuarcita y tres de ftanita). Uno de los huesos presenta huellas de procesamiento humano, lo cual confirma el origen arqueológico de los mismos.

\section{Representación anatómica y estructura etaria de vizcacha}

Sobre la base de la lateralidad, tamaño y fusión ósea de los elementos de la muestra se estimó un número mínimo de tres vizcachas. Estas corresponden a las categorías etarias de neonato, juvenil y subadulto. Los elementos mejor representados, con un Ri entre 76 y $100 \%$, son la hemimandíbula y la tibia. Estos son seguidos por el cráneo, la pelvis y el fémur (51 a 75\%). En tercer lugar, se encuentran la escápula, el sacro, el húmero, el radio, el calcáneo y la vértebra lumbar (26 a 50\%). Finalmente, los metapodios y costillas están escasamente representados (menos de $25 \%$ ). Es importante mencionar que varias unidades anatómicas como vértebra cervical, vértebra torácica, clavícula, ulna, astrágalo, articulares y falanges se encuentran ausentes (Tabla 4).

\section{Análisis tafonómico de los restos óseos}

La observación de la superficie cortical de los huesos que pudieron ser asignados a una categoría taxonómica $(\mathrm{N}=161)$ evidencia que los surcos constituyen el efecto tafonómico mejor representado en la muestra ( $\mathrm{N}=67$, $41,6 \%$ ) (Tabla 5). El taxón con mayor frecuencia de surcos fue la vaca $(\mathrm{N}=49,70 \%)$, seguida por el guanaco $(\mathrm{N}=3$, $42,8 \%)$, la vizcacha $(\mathrm{N}=13,32,5 \%)$ y el zorro pampeano $(\mathrm{N}=1,2,8 \%)$. Los daños que generaron los carnívoros también fueron abundantes $(\mathrm{N}=34,21,1 \%)$. Entre los taxones más afectados se encuentra la vaca ( $\mathrm{N}=22$, $31,4 \%)$, seguida por la vizcacha $(\mathrm{N}=5,12,5 \%)$ y el zorro pampeano ( $\mathrm{N}=3,8,3 \%)$. Es importante mencionar que los taxones con escasa representación en la muestra, como Anura, Strigiformes y Paseriformes, se encontraron en su mayoría afectados por depredadores. La meteorización se observa solo en restos de vaca $(\mathrm{N}=25,35,7 \%)$, guanaco $(\mathrm{N}=5,71,4 \%)$ y vizcacha $(\mathrm{N}=2,5 \%)$, con estadios bajos. Tres elementos óseos de vaca $(4,3 \%)$ exhibieron efectos compatibles con la osteofagia generada por ungulados. La presencia de líquenes se registra en pocos especímenes de vaca $(\mathrm{N}=8,11,4 \%)$ y vizcacha $(\mathrm{N}=1,2,5 \%)$. El guanaco es el único taxón que presenta especímenes óseos

\begin{tabular}{|c|c|c|c|c|}
\hline Elemento & NISP & MNE & MNI & Ri \\
\hline Cráneo (1) & 2 & 2 & 2 & 66,66 \\
\hline Hemimandibula (2) & 5 & 5 & 3 & 83,33 \\
\hline V. Lumbar (5) & 5 & 5 & 1 & 23,80 \\
\hline Sacro (1) & 1 & 1 & 1 & 33,33 \\
\hline Costilla (24) & 4 & 4 & 1 & 5,55 \\
\hline Escápula (2) & 3 & 3 & 2 & 50 \\
\hline Húmero (2) & 1 & 1 & 1 & 33,33 \\
\hline Radio (2) & 1 & 1 & 1 & 33,33 \\
\hline Hemipelvis (2) & 5 & 4 & 3 & 66,66 \\
\hline Fémur (2) & 4 & 4 & 3 & 66,66 \\
\hline Tibia (2) & 5 & 5 & 3 & 83,33 \\
\hline Calcáneo (2) & 2 & 2 & 1 & 33,33 \\
\hline Metapodio (14) & 2 & 2 & 1 & 4,76 \\
\hline
\end{tabular}

Tabla 4. MNE, MNI y Ri de vizcacha.

Tabla 4. MNE, MNI, and y Ri of vizcacha. 


\begin{tabular}{|ll|c|c|c|c|c|c|c|c|c|}
\hline Taxón & & 1 & 2 & 3 & 4 & 5 & 6 & 7 & 8 & 9 \\
\hline Meteorización & 0 & 64,3 & 95 & 100 & - & 100 & 100 & 100 & 100 & 100 \\
\hline & 1 & 34,3 & 5 & - & 71,4 & - & - & - & - & - \\
\hline & 2 & 1,4 & - & - & - & - & - & - & - & - \\
\hline Carnívoros & 3 & - & - & - & 28,6 & - & - & - & - & - \\
\hline Osteofagia & 31,4 & 12,5 & 8,3 & - & - & 100 & 66,7 & 100 & - \\
\hline Surcos & 4,3 & - & - & - & - & - & - & - & - \\
\hline Liquenes & 70 & 32,5 & 2,7 & 42,8 & 100 & - & - & - & - \\
\hline Manganeso & 11,4 & 2,5 & - & - & - & - & - & - & - \\
\hline Huellas de corte & - & - & - & 14,3 & - & - & - & - & - \\
\hline
\end{tabular}

Tabla 5. Variables tafonómicas registradas en la muestra ósea.

Tabla 5. Taphonomic variables recorded in the bone sample.

Referencias: $1=B$. taurus; $2=L$. maximus; $3=$ L. gymnocercus; $4=$ L. guanicoe; $5=R$. americana; $6=$ Strigiformes; $7=$ Passeriformes; $8=$ Anura y $9=$ Ave pequeña indeterminable.

con tinción por manganeso $(\mathrm{N}=1,14,3 \%)$ y huellas de procesamiento antrópico $(\mathrm{N}=1,14,3 \%)$.

Los surcos presentes en los huesos de vaca

Se realizó el estudio detallado de los surcos presentes en los huesos de vaca, ya que este taxón es el que exhibe la mayor frecuencia de este efecto. El análisis realizado informa que tres de las cuatro vizcacheras estudiadas presentaban huesos con estas modificaciones. Se registraron surcos en todos los elementos que componen el esqueleto, aunque el porcentaje de las vértebras afectadas fue el menor (47,8\%). En 31 (63,3\%) elementos, la frecuencia de estas marcas es baja (menos de 10 por espécimen), en $13(26,5 \%)$ media (entre 10 y 50 por espécimen) y en $5(10,20 \%)$ alta (más de 50 por espécimen). Los elementos que generalmente presentaron frecuencias menores son las vértebras, en tanto que el cráneo, escápula, pelvis y huesos largos mostraron las más altas.

Los surcos generalmente se ubican en las diáfisis de los huesos largos y costillas, en las apófisis transversas de las vértebras y en los sectores prominentes del cráneo (e.g., arco zigomático). La pelvis presenta mayor concentración de marcas en el cuello del íleon y las escápulas muestran una distribución radial de los surcos sobre los bordes de la hoja. Una de las escápulas mostró pérdida ósea en uno de los vértices de su hoja, asociada con múltiples surcos anchos (Figura 5).

Los surcos generalmente se orientan de manera transversal al eje longitudinal del hueso $(77,5 \%)$ y en menor medida lo hacen en forma oblicua $(28,6 \%)$ y longitudinal $(6,1 \%)$. La diversidad de estas modificaciones fue clasificada en dos grandes grupos. Por un lado, los surcos anchos (Figura 6). Estos son poco profundos, de base plana con numerosas estrías longitudinales en su interior. En algunos casos se observan extrusiones que destacan entre las estrías y eventualmente pueden ubicarse en la mitad del rasgo. Estos surcos pueden presentarse rectos, levemente curvos y sinuosos. Se manifiestan aislados o asociados en pequeños grupos. Se registraron largos máximos de

Figura 5. Detalle de escápula de vaca (ET.CV4.1) donde se puede observar numerosos surcos anchos asociados a pérdida ósea.

Figure 5. Detail of cow scapula (ET. CV4.1) where several wide grooves associated to bone loss can be observed.

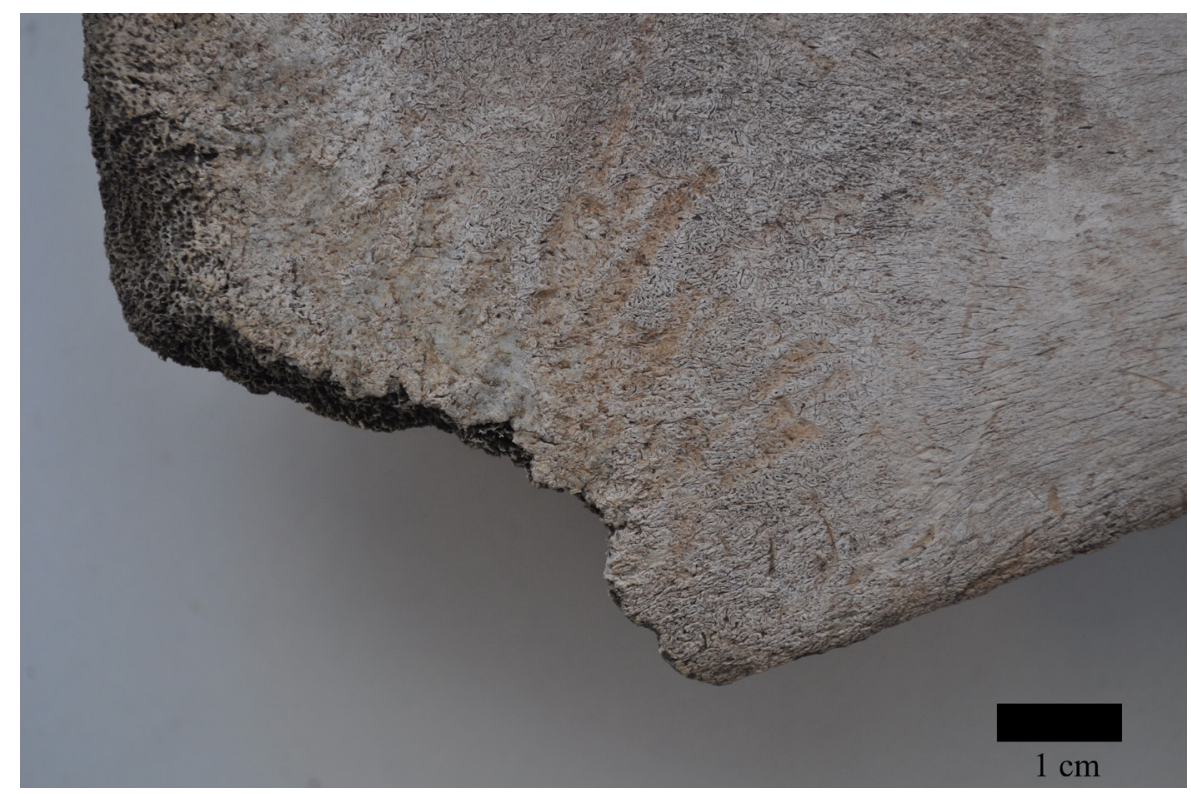


$42,98 \mathrm{~mm}$ y anchos que llegan a 4,2 $\mathrm{mm}$. Por otro lado, los surcos finos (Figura 7) pueden ser superficiales 0 profundos de sección en $\mathrm{V}$ o en $\mathrm{U}$. En algunas ocasiones aparecen de sección en $\mathrm{V}$ con una inclinación de $45^{\circ}$. Generalmente, no se observan estrías en su interior y en algunos casos se registran dobles paralelas o simples con bifurcación. Su morfología más común es curvada, aunque pueden presentarse rectas o sinuosas. Sus largos más frecuentes varían entre 5 y $15 \mathrm{~mm}$.

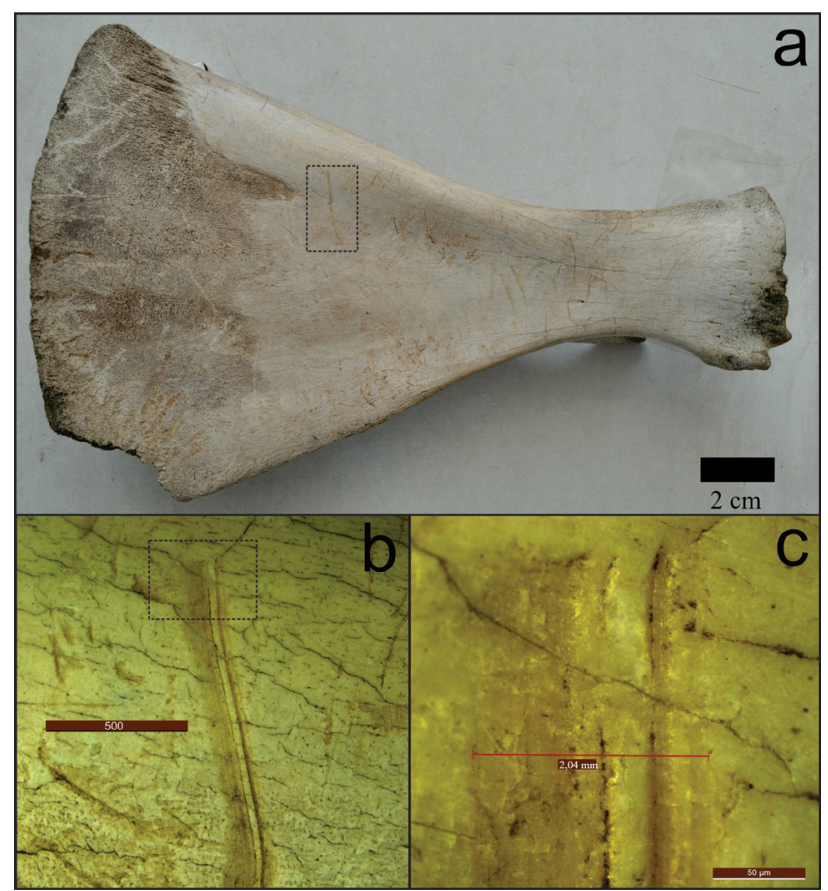

Un 95,9\% de los elementos presentaba al menos un surco de tipo fino y un $22,4 \%$ al menos uno de tipo ancho. Cabe aclarar que varios de los elementos exhibían ambos tipos de modificaciones y en un caso se observó que un surco comenzaba ancho y terminaba fino.

\section{Discusión}

Este estudio actualista complementa los trabajos previos sobre vizcacha de la llanura, que indican que estos roedores generan un impacto significativo en las áreas que ocupan (Branch et al. 1996; Campos 1997; Arias et al. 2003, 2005; Curetti 2007; Rafuse et al. 2019). Este mamífero constituiría, junto con los armadillos, unos de los principales agentes biológicos perturbadores de los sitios arqueológicos de la región pampeana (Salemme et al. 2012; Álvarez et al. 2020). A diferencia de los armadillos, que ocupan el paisaje de manera homogénea, las vizcacheras se asientan sobre lugares bien drenados cercanos a lagunas y cursos de agua, superponiéndose en gran medida con las ocupaciones humanas del pasado. De hecho, este estudio evidenció que un $47,6 \%$ de las vizcacheras presentaba restos arqueológicos asociados con los sedimentos removidos por los roedores. Estas vizcacheras se ubican en distintos sectores del borde de la laguna y muestran el potencial de ocupaciones humanas en este sector. El impacto que las vizcachas generan sobre los sitios arqueológicos implica diversos aspectos que se discuten a continuación.

Figura 6. A) Vista medial de una escápula de vaca (ET.CV4.1) con presencia de numerosos surcos. El recuadro corresponde al sector donde se encuentra un surco ancho sobre el cual tomaron fotografías con mayores aumentos. B y C) surco bajo aumento de lupa binocular.

Figure 6. A) Medial view of cow scapula (ET. CV4.1) with presence of several grooves. The square corresponds to the section with a wide groove where detailed photographs were taken; $B$ and $C$ ) groove under magnification.

Figura 7. Vista lateral de la escápula de vaca (ET. CV4.1). El recuadro muestra el sector donde se halla el surco fino de sección en $\mathrm{V}$. B y C) surco bajo aumento de lupa binocular.

Figure 7. Lateral view of cow scapula (ET.CV4.1). The square shows a thin groove with $V$-section; $B$ and C) groove under magnification.

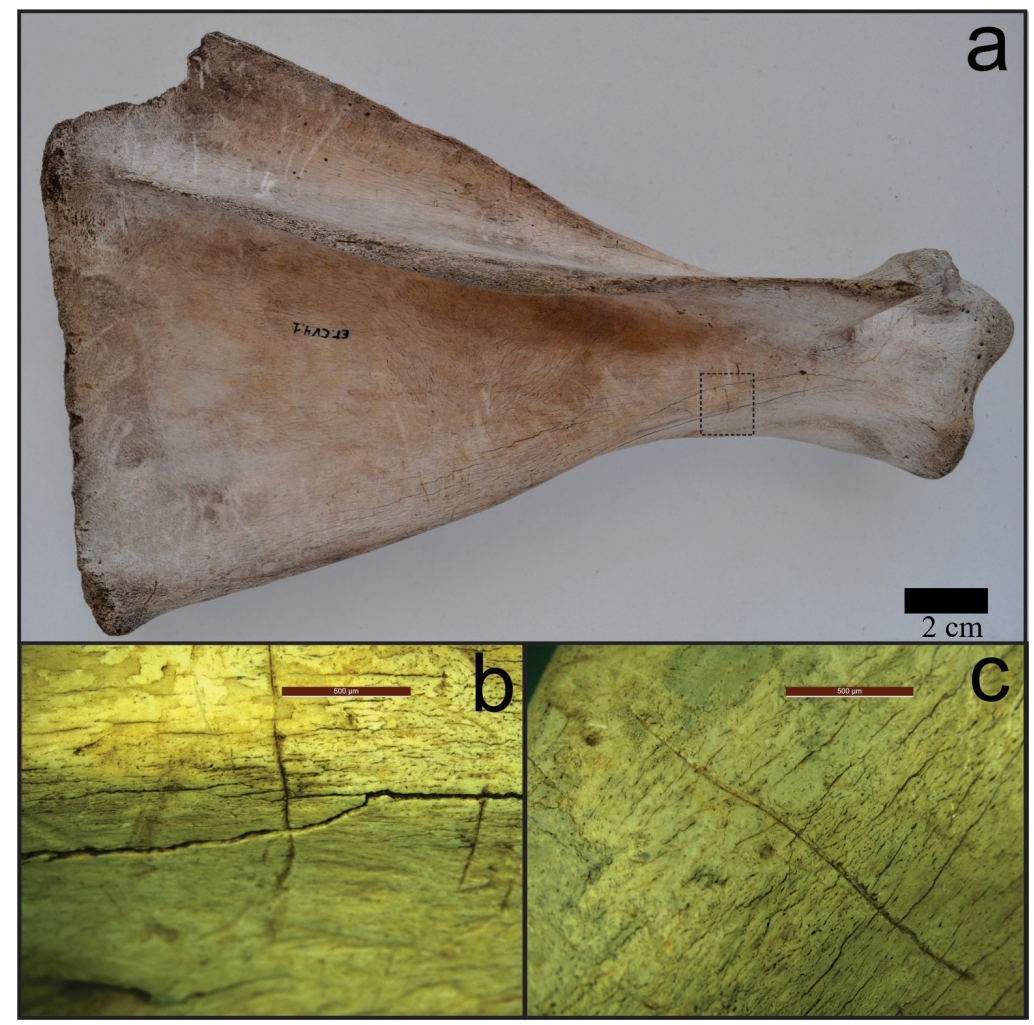


Las vizcachas alteran los sedimentos mediante la realización de sus cuevas, ocasionando una fuerte perturbación de los suelos

A partir de este estudio se registraron 21 vizcacheras que afectaron un área de $1226 \mathrm{~m}^{2}$. Por otra parte, Rafuse et al. (2019) reportan 32 vizcacheras, con un área afectada de $4730 \mathrm{~m}^{2}$. Ambos registros alertan acerca de la extensa área que pueden modificar estos roedores. Además, los sistemas de vizcacheras permanecen activos durante largos períodos de tiempo y algunos autores han relevado un uso continuo de las madrigueras de hasta setenta años (Weir 1974). La construcción y mantenimiento de los sistemas de madrigueras genera la redistribución vertical y horizontal de materiales y sedimentos. La reiteración de este proceso altera las secuencias estratigráficas naturales y arqueológicas, dando como resultado la presencia de biofábricas con mezcla de materiales de distinto origen y cronología (Bellinzoni y Kaufmann 2014; Rafuse et al. 2019). En este sentido, puede mencionarse el ejemplo del sitio Laguna Muscar 2, en el cual la acción de las vizcachas produjo el movimiento de diversos materiales arqueológicos y de un enterratorio humano (Kaufmann et al. 2019). En los niveles inferiores del sitio, los roedores generaron el desplazamiento de los pies, manos, columna vertebral, costillas y miembros delanteros de un esqueleto datado en ca. 2500 años AP. Asimismo, esta acción produjo el traslado de elementos óseos por fuera del área excavada y afectó la completitud del esqueleto. Estos procesos también habrían provocado la fractura de los elementos más frágiles del esqueleto, como costillas, vértebras y escápulas (Kaufmann et al. 2019). Los niveles superiores, suprayacentes al enterratorio también fueron perturbados, lo que dio origen a asociaciones de materiales de diversa cronología, como fauna pleistocénica, huesos de guanaco y fauna europea.

Las vizcacheras son visitadas y ocupadas por diversos agentes bióticos, dando lugar a conjuntos faunísticos de alta diversidad taxonómica

Las vizcacheras constituyen ambientes protegidos y con características ideales para ser ocupadas por aves, anuros y ofidios (Pocheville 2015). En este estudio se relevaron evidencias directas e indirectas de la ocupación de lechucitas de las vizcacheras y de la visita recurrente de pequeños carnívoros como zorros y hurones (estos animales fueron observados durante los trabajos de campo), indudablemente atraídos por presas potenciales y carroña. La alta riqueza taxonómica registrada a través de los restos óseos constituye un fiel reflejo de las interacciones ecosistémicas complejas, las cuales se sumarían a las propias de un sitio arqueológico. Al respecto, en el caso del sitio Laguna Muscar 2, el conjunto faunístico presenta una elevada riqueza taxonómica en la que dominan ampliamente los huesos de vizcacha (Bellinzoni 2018). Las estructuras subterráneas podrían haber actuado como trampas, favoreciendo la acumulación de restos de vertebrados pequeños y microvertebrados muertos por causas eto-ecológicas o por la acción de depredadores (Behrensmeyer y Hook 1992; Pardiñas 1999; Tomassini et al. 2019).

El NISP\% de vizcachas se encuentra entre los más elevados en un contexto de vizcacheras

Las vizcachas constituyen el segundo taxón mejor representado de la muestra con un número de restos de 40 y un NISP\% de 15,3. Los elementos más frecuentes son la hemimandíbula y la tibia. En general, los huesos largos y el cráneo se encuentran bien representados, en tanto que para el esqueleto axial se registran valores más bajos, con elementos ausentes. Entre estos últimos se destacan los huesos pequeños, como los articulares y las falanges. Este perfil de partes se corresponde con aquellos usualmente registrados para los conjuntos atricionales afectados por procesos mediados por la densidad mineral. Gutiérrez et al. (2016) reportaron patrones similares para los conjuntos óseos de liebre procedentes de muestras naturalistas, así como para restos experimentales resultado de la alimentación de pequeños carnívoros con conejos (Álvarez et al. 2012; Rafuse et al. 2014; Massigoge et al. 2014; Scheifler et al. 2020). En relación con la ausencia de los huesos pequeños, es importante considerar que estos son más susceptibles a la destrucción, pero también al rápido entierro (Beherensmeyer y Boaz 1980; Beherensmeyer y Chapman 1993).

Un estudio similar al presentado en este trabajo fue realizado por Rafuse et al. (2019). Estos autores determinaron un elevado MNI (31), el cual podría estar vinculado con episodios de muertes por causa humana (envenenamiento). En líneas generales, existen coincidencias entre el perfil de partes esqueletarias obtenido en la muestra superficial analizada por Rafuse et al. (2019) y el presentado en este estudio. De acuerdo con los investigadores mencionados, el conjunto de superficie pudo haber sido depositado de forma atricional. Este fue comparado con perfiles generados por diferentes depredadores y se obtuvieron correlaciones estadísticas positivas con el águila real, el gato montés y el zorro pampeano. Este aspecto está vinculado con que en ambos conjuntos actuaron los carnívoros, lo cual es evidenciado por la alta frecuencia de marcas de dientes $(8,4 \%$ en Rafuse et al. 2019 y 21,1\% en este estudio).

Las vizcachas acarrean diversos objetos que se encuentran disponibles localmente al entorno de sus madrigueras

Nuestro estudio confirma observaciones previas respecto al hábito acumulador que tiene este roedor (Rafuse et al. 2019; Tomassini et al. 2019). En el $85 \%(n=18)$ de las unidades de análisis se registraron objetos como tallos secos, toscas, huesos, bosta seca, metales, plásticos, goma y vidrio. En general, los huesos transportados fueron especímenes de vaca que tenían grados de meteorización leves. Las uniones óseas y el peso de los huesos y toscas permitieron estimar un promedio 
de distancia de transporte de 33,1 m, con un máximo de $40,3 \mathrm{~m}$ y pesos promedios de $338,2 \mathrm{gr}$ con un máximo de 1583 gr. Este comportamiento acumulador también es característico de los puercoespines (Hystrix africaeaustralis, Hystrix indica) (Alexander 1956; Dart 1957; Brain 1981; Kibii 2009) y de roedores más pequeños del género neotoma (Hoffman y Hays 1987; Hockett 1989). En general, la diversidad y dimensiones de objetos transportados parece ser una función del tamaño de los roedores y la disponibilidad local de materiales. El acarreo de huesos secos y levemente meteorizados a sus madrigueras se ha relacionado principalmente con el mantenimiento de sus dientes y, alternativamente, con la osteofagia (Lyman 1994).

A través de su comportamiento, las vizcachas producen parches en los cuales la composición, biomasa y niveles de nutrientes de la vegetación es diferencial

El forrajeo, la constante depositación de heces y el acarreo de rocas calizas contribuyen a concentrar fósforo $(P)$ en los suelos cercanos a las cuevas. Esto favorece la existencia de parches de vegetación sobre las vizcacheras (Villarreal et al. 2008). A través de este proceso, las vizcachas afectan directa e indirectamente la disponibilidad de recursos para otras especies y actúan como ingenieros de ecosistemas, modificando, manteniendo y/o creando nuevos hábitats (Jones et al. 1994; Villarreal et al. 2008). Esto aún no ha sido explorado en casos arqueológicos, pero su análisis a partir de estudios de suelo podría constituir una vía de entrada a futuro.

Al roer los huesos las vizcachas generan marcas que las diferencian de otros agentes

Las vizcachas, así como otros roedores, lagomorfos y algunas especies de marsupiales (Vombatus ursinus) y ungulados (Vicugna vicugna) poseen incisivos de crecimiento continuo (Oshima et al. 2005; Madden 2015; Tapaltsyan et al. 2015). La altura funcional de estos dientes se mantiene naturalmente a través de la proliferación celular de los extremos apicales y el desgaste de los bordes incisales generado por la alimentación (Oshima et al. 2005). Cuando la tasa de desgaste natural de los incisivos resulta menor a la del crecimiento, estos animales mantienen el equilibrio funcional de sus dientes royendo diversos objetos (Brain 1981). Otra razón propuesta de este roído es la obtención de los minerales contenidos en los huesos (Brain 1981; Haglund et al. 1988; Thornton y Fee 2001). Los estudios tafonómicos indican que los roedores pueden generar una gran diversidad de marcas en función del tamaño de la especie (Mengoni Goñalons 1999; Tong et al. 2008). Diversas situaciones como la densidad y el estado de los huesos (frescos-secos), en combinación con el ángulo y fuerza de la mordida, inclinación e integridad de los incisivos pueden otorgar variabilidad en las marcas generadas (Politis y Madrid 1988; Quintana 2007; Tong et al. 2008).
La frecuencia de huesos con evidencias de surcos provenientes de las vizcacheras $(41,6 \% ; n=67)$ es acorde con el registro obtenido en diversos conjuntos modificados por roedores grandes como los histricomorfos. Los porcentajes de huesos afectados oscilan entre el 20 y $100 \%$, con promedios de 52,3\% (Maguire 1976; Brain 1981). No obstante, la intensidad del daño que observamos en los huesos roídos por vizcacha es menor a la que reportan los autores citados. Los roedores concentrarían su roído sobre huesos secos de mamíferos grandes. Además, también podrían actuar sobre epífisis poco densas en huesos frescos, generando el clásico scooping out (ahuecado) descripto para carnívoros (Maguire et al. 1980, Eickoff y Hernan 1985; Klippel y Synstelien 2007). Las marcas que inequívocamente pudimos atribuir a vizcachas fueron clasificadas en surcos anchos de fondo plano y poco profundos con estrías en su interior, y surcos finos, más profundos con sección en $\mathrm{V}$ o en $U$. Ambos grupos de marcas poseen largos que oscilan entre 5 y $15 \mathrm{~mm}$, con largos máximos de $43 \mathrm{~mm}$. Estas, generalmente se orientan de manera transversal al eje longitudinal del hueso y pueden ser curvas, sinuosas o rectas. Su tamaño se diferenciaría de las generadas por roedores pampeanos pequeños como cricétidos (Quintana 2007) y serían similares a las producidas por los coipos (Myocastor coypus) (Politis y Madrid 1988).

\section{Conclusiones}

La vizcacha de la llanura ha estado presente en la región pampeana desde el arribo de los primeros pobladores, hace más de 12.000 años, hasta la actualidad. Este animal fue aprovechado para el consumo de su carne, cuero y huesos. Paralelamente, la vizcacha ha intervenido en los procesos de formación de diversos sitios arqueológicos de la región (Salemme y Madrid 2007; Lanzelotti y Bonaparte 2009; Salemme et al. 2012; Politis 2014; Kaufmann et al. 2019). Por este motivo, es importante conocer la forma en que este chinchílido altera los sitios arqueológicos en la actualidad y considerar que dichas perturbaciones también tuvieron lugar en el pasado.

La vizcacha genera impactos en diferentes escalas, que podrían afectar la resolución e integridad de localidades arqueológicas o sitios y de conjuntos y especímenes óseos. Este roedor altera las secuencias estratigráficas de grandes áreas del paisaje a través del movimiento y mezcla de sedimento y materiales que se encontraban en diferentes niveles o estratos. En ocasiones, estas cuevas pueden preservar su morfología original y rellenarse con sedimentos y materiales diversos, y en otras, pueden colapsar o ser obliteradas por la redundancia de excavaciones a lo largo del tiempo. Este último caso daría lugar a biofábricas que pueden ocupar importantes porciones y áreas de las secuencias estratigráficas. Además, este roedor acarrea materiales disponibles localmente al entorno de sus madrigueras, 
aspecto que genera nuevas asociaciones de materiales que resultarán muy complejas de interpretar. En este trabajo pudimos registrar huesos que fueron movidos por distancias de hasta 40,3 m y con pesos de hasta un kilo y medio. Por otra parte, la vizcacha también altera los sedimentos químicamente, lo cual genera parches en el paisaje con una química y vegetación particular. En lo que respecta a la integridad de los conjuntos faunísticos, las vizcacheras constituyen nichos ecológicos que son ocupados y visitados por diversos organismos, dando lugar a conjuntos faunísticos de alta diversidad taxonómica. Otra característica de estos ensambles es la alta frecuencia de restos de vizcacha, consecuencia de las muertes eto-ecológicas o por depredación. Estos restos corresponderán a individuos con diverso desarrollo ontogénico y los perfiles de partes esqueletarias estarán usualmente afectados por la acción de carnívoros. A nivel de los especímenes óseos, las vizcachas generan marcas que permiten diferenciarlas de la acción de otros agentes. En este estudio se observó que estos roedores concentran su roído mayormente sobre huesos secos de mamíferos grandes, pudiendo generar, además de surcos, pérdida ósea en algunos elementos. En este caso, un resultado importante de este trabajo es que si se considera la variabilidad de tamaños de roedores de la región pampeana sería posible diferenciar las marcas de incisivos de grupos pequeños (cricétidos y cávidos) y grandes (coipo, vizcacha y carpincho).

Finalmente, este estudio realiza un aporte en relación con la caracterización de animales de hábitos fosoriales que se ha venido desarrollando en la región pampeana (Frontini y Deschamps 2007; Frontini y Escosteguy 2012; Rafuse et al. 2019; Tomassini et al. 2019; Álvarez et al. 2020). Estos estudios indican que los armadillos y las vizcachas son los animales cavadores que perturban con mayor intensidad los sitios arqueológicos. Los armadillos lo harían de forma relativamente homogénea en el paisaje, en tanto que las vizcachas se concentrarían en lugares altos y cercanos a cuerpos y cursos de agua. La acción de este roedor sería más fácil de distinguir. A diferencia del contexto de las cuevas de armadillos, generalmente en el de las vizcachas hay alta frecuencia de sus huesos y marcas de roído sobre los restos óseos. El conocimiento minucioso de las alteraciones producidas por los animales de hábitos fosoriales permitirá evaluar con mayor cautela el registro arqueológico del área.

Olavarría, 19 de mayo de 2020

\section{Agradecimientos}

Esta investigación fue subsidiada por los proyectos PICT 2015-0235 y PICT 2017-1969. La UE-INCUAPA-CONICET y la Facultad de Ciencias Sociales (UNICEN) nos facilitó los medios para realizar los trabajos de campo y almacenar las colecciones. Queremos agradecer al señor Fernández y a la familia Rivera, quienes nos permitieron trabajar en sus establecimientos. Finalmente, queremos agradecer a la Dra. Romina Frontini por las sugerencias en la versión preliminar de este trabajo y a dos evaluadores anónimos por sus valiosos aportes.

\section{Bibliografía}

Alexander, A. J. (1956). Bone carrying by a porcupine. South African Journal of Science, 52, 257-258.

Álvarez, M. C. (2009). Análisis de los restos faunísticos del sitio Calera (Sierras Bayas, Partido de Olavarría). Un aporte a su funcionalidad a través del estudio de los mamíferos. Relaciones de la Sociedad Argentina de Antropología, XXXIV, 29-51.

Álvarez, M. C. (2014). Subsistence patterns during the Holocene in the Interserrana area (pampean region, Argentina): evaluating intensification in resource exploitation. Journal of Anthropological Archaeology, 34, 54-65.

Álvarez, M. C., Kaufmann, C. A., Massigoge, A., Gutiérrez, M. A., Rafuse, D. J., Scheifler, N. y González, M. E. (2012). Bone modification and destruction patterns of leporid carcasses by Geoffroy's cat (Leopardus geoffroyi): an experimental study. Quaternay International, 278, 71-80.

Álvarez, M. C., Alcaraz, A. P., Gutiérrez, M. A. y Martínez, G. A. (2013). Análisis zooarqueológico del sitio Paso Otero 4 (partido de Necochea). Aportes a la discusión de los modelos de subsistencia de la región pampeana. Intersecciones en Antropología, 14, 383-398.

Álvarez, M. C., Rafuse, D., Bellinzoni, J. y Kaufmann, C. A. (2020). Armadillos as taphonomic agents of archaeological sites in the Pampas Region, Argentina. Journal of Archaeological Science: Reports, 31, 102293.

Andrews, P. (1990). Ow/s, Caves and Fossils. Londres: The University of Chicago Press.

Arias, S. M., Madanes, N. y Quintana, R. D. (2003). Estructura y composición de la vegetación en vizcaheras activas e inactivas en el Delta del Paraná. Mastozoología Neotropical, 10, 9-20.

Arias, S. M., Quintana, R. D. y Cagnoni, M. (2005). Vizcacha's influence on vegetation and soil in a wetland of Argentina. Rangeland Ecology \& Management, 58, 51-57.

Balek, C. L. (2002). Buried artifacts in sable upland sites and the role of bioturbation: A review. Geoarcheology, $17,41-51$

Barone, R. (1999). Anatomie Comparée des Mammifères 


\section{Domestiques. París: Vigot Frères.}

Barros, M. P., Bellinzoni, J. y Kaufmann, C. A. (2018a). Análisis de los instrumentos líticos de los sitios Laguna La Redonda y Laguna Muscar 2 (Partido de Gral. Lamadrid, provincia de Buenos Aires). Intersecciones en Antropología, 19(2), 111-122.

Barros, M. P., Heider, G., Álvarez M. C., Kaufmann, C. y Bellinzoni, J. (2018b). First results of the hunter-gatherer weapon system studies in the middle basin of the Salado creek (Pampas Region, Argentina). Journal of Lithic Studies, 5, 1-23.

Barros, M. P., Bellinzoni, J., Colantonio, M. J., Álvarez, M. C. y Kaufmann, C. A. (2020). Aportes del sitio Hangar al estudio de la gestión tecnológica en la cuenca del Arroyo Salado (provincia de Buenos Aires) durante el Holoceno tardío. Revista del Museo de Antropología, 13(1), 237-242.

Behrensmeyer, A. K. (1978). Taphonomic and ecologic information from bone weathering. Paleobiology, 4, 150-162.

Behrensmeyer, A. K. y Hook, R. W. (1992). Paleoenvironmental contexts and taphonomic modes. En A. K. Behrensmeyer, J. D. Damuth, W. A. DiMichele, R. Potts, H-D. Sues y S. L. Wing (Eds.), Evolutionary Paleoecology of Terrestrial Plants and Animals (pp. 15136). Chicago: University of Chicago.

Behrensmeyer, A. K. y Boaz, D. E. (1980). The recent bones of Amboseli National Park, Kenya. En A. K. Behrensmeyer y A. Hill (Eds.), Fossils in the Making (pp. 72-92). Chicago: University of Chicago Press.

Behrensmeyer, A. K. y Chapman, R. (1993). Models and simulations of time-averaging in terrestrial vertebrate accumulations. En S. M. Kidwell y A. K. Behrensmeyer (Eds.), Taphonomic Approaches to Time Resolution in Fossil Assemblages. Short Courses in Paleontology 6 (pp. 125-149). Knoxville: Paleontological Society.

Bellinzoni, J. (2018). Análisis Zooarqueológico y Tafonómico del Sitio Laguna Muscar 2 (Pdo. De General Lamadrid, Pcia de Buenos Aires). Tesis de Licenciatura Inédita. Olavarría: Universidad Nacional del Centro de la Provincia de Buenos Aires.

Bellinzoni, J. y Kaufmann, C. A. (2014). Las vizcachas (Lagostomus maximus) como agentes tafonómicos. En Libro de resúmenes del Séptimo Congreso de Arqueología de la Región Pampeana Argentina (p. 96). Rosario: Ministerio de Innovación y Cultura del Gobierno de Santa Fe.

Binford, L. R. (1981). Bones: Ancient Men and Modern
Myths. Nueva York: Academic Press.

Bonomo, M., Leon, D. C., Turnes, L. y Apolinaire, E. (2008). Nuevas investigaciones sobre la ocupación prehispánica de la costa pampeana en el Holoceno tardío: el sitio arqueológico Claromecó 1 (partido de Tres Arroyos, provincia de Buenos Aires). Intersecciones en Antropología, 9, 25-41.

Brain, C. K. (1981). The Hunters or the Hunted. Chicago: University of Chicago Press.

Branch, L. y Sosa, A. (1994). Foraging behaviour of the plains vizcacha (Lagostomus maximus, Family Chinchillidae). Vida Silvestre Neotropical, 3(2), 9699.

Branch, L. C., Villarreal, D. y Fowler, G. S. (1993). Recruitment, dispersal, and group fusion in a declining population of the Plains vizcacha (Lagostomus maximus, chinchillidae). Journal of Mammalogy, 74, 9-20.

Branch, L. C., Villarreal, D., Pessino, M. E. M. y Villarreal, D. (1996). Response of pumas to a population decline of the Plains vizcacha. Journal of Mammalogy, 77, 1132-1140.

Cabrera, A. (1961). Catálogo de los mamíferos de América del Sur. Parte II. Revista del Museo Argentino de Ciencias Naturales "Bernardino Rivadavia" Ciencias Zoológicas, 4, 309-732.

Cáceres, I., Esteban-Nadal, M., Bennasar, M., Marín Monfort, M. D., Pequero, M. D. y Fernández-Jalvo, Y. (2013). Osteophagia and dental wear in herbivores: actualistic data and archaeological evidence. Journal of Archaeological Science, 40, 3105-3116.

Campos, C. M. (1997). Utilización de recursos alimentarios por mamíferos medianos y pequeños del desierto del Monte Tesis Doctoral Inédita. Córdoba: Universidad Nacional de Córdoba.

Cirignoli, S. y Lartigau, B. (2019). Lagostomus maximus. En SAyDS-SAREM (Eds.), Categorización 2019 de los mamíferos de Argentina según su riesgo de extinción. Lista Roja de los mamíferos de Argentina. Versión digital. http://cma.sarem.org.ar. Accedido el 14 de mayo de 2020.

Curetti, P. (2007). Tafonomía en movimiento: Las vizcacheras del Alizal (Alejandra, Santa Fe). En F. Oliva, N. de Grandis y J. Rodríguez (Eds.), Arqueología Argentina en los Inicios de un Nuevo Siglo, Tomo I ( pp. 573-577). Rosario: Laborde Editor.

Dart, R. A. (1957). The osteodontokeratic culture of Australopithecus Prometheus. Transvaal Museum 
Memoir, 10. Pretoria.

Eickoff, S. y Hernan, B. (1985). Surface marks on bones from a Neolithic collective grave (Odagsen, lower Saxony): a study on differential diagnosis. Journal of Human Evolution, 14, 263-274.

Escosteguy, P. y Salemme, M. (2012). Butchery evidence on rodent bones from archaeological sites in the Pampean Region (Argentina). En. C. Lefèvre (Ed.), Proceedings of the General Session of the 11th ICAZ International Conference (Paris, 23-28 August 2010) (pp. 227-237). Oxford: B.A.R. (International Series) 2354. Archaeopress.

Fisher, J. (1995). Bone surface modifications in zooarchaeology. Journal of Archaeological Method and Theory, 2(1), 7-68.

Frontini, R. y Deschamps, C. (2007). La actividad de Chaetophractus villosus en sitios arqueologicos. El Guanaco como caso de estudio. En C. Bayón, A. Pupio, M. I. González, N. Flegenheimer y N. M. Frere (Eds.), Arqueología en las Pampas (pp. 439-451). Buenos Aires: Sociedad Argentina de Antropología.

Frontini, R. y Escosteguy, P. (2012). Chaetophractus villosus: A disturbing agent for archaeological contexts. International Journal of Osteoarchaeology, 22, 603615.

Getty, R., Sisson, S. y Grossman, J. D. (1982). Anatomía de los Animales Domésticos. Barcelona: Salvat.

Giulietti, J. D. y Veneciano, J. H. (2005). La vizcacha. Informativo Rural, E.E.A INTA San Luis, 2(7), 8-10.

Gutiérrez, M. A., Kaufmann, C. A., González, M. E., Scheifler, N. A., Rafuse, D. J., Massigoge, A. y Álvarez, M. C. (2016). The role of small carnivores in the movement of bones: implications for the Pampas archaeofaunal record, Argentina. Archaeological and Anthropological Science, 8, 257-276.

Haglund, W. D., Reay, D. T. y Swindler, D. R. (1988). Tooth mark artifacts and survival of bones in animal scavenged human skeletons. Journal of Forensic Sciences, 33, 985997.

Haynes, G. (1980). Evidence of carnivore gnawing on Pleistocene and recent mammalian bones. Paleobiology, 6, 341-351.

Hockett, B. S. (1989). The concept of "carryng range:" a method for determining the role played by woodrats in contributing bones to archaeological sites. Nevada archaeologist, 7, 28-35.
Hoffman, R. y Hays, C. (1987). The Eastern Wood rat (Neotoma Floridana) as a taphonomic factor in archaeological sites. Journal of Archaeological Science, $14,325-337$.

Jackson, J. E. (1986). Determinación de edad en la vizcacha (Lagostomus maximus) en base al peso del cristalino. Vida Silvestre Neotropical, 1(1), 41-44.

Jackson, J. E., Branch, L. C., Villarreal, D., Inserra, P. I. F., Jackson, J. E., Branch, L. C. y Villarreal, D. (1996). Lagostomus maximus. Mammalian Species, 543, 1-6.

Jofré, G. (1994). Selección de forraje por vizcacha (Lagostomus maximus) en un área degradada del chaco occidental de San Luis. Seminario de Licenciatura en Ciencias Biológicas. San Luis: Universidad Nacional de San Luis.

Johnson, D. L. (1990). Biomantle evolution and the redistribution of earth materials and artifacts. Soil Science, 149, 84-102.

Johnson, D. L. (2002). Darwin would be proud: Bioturbation,dynamic denudation, and the power of theory in science. Geoarchaeology, 17, 7-40.

Jones, C. G., Lawton, J. H. y Shachak, M. (1994). Organisms as ecosystem engineers. Oikos, 69, 373386.

Kaufmann, C. A. y González, M. (2013). Rescate arqueológico de restos óseos humanos en el sitio Laguna Seca 1 (Pdo. de Gral. Lamadrid, Pcia. de Buenos Aires). Revista del Museo de la Plata, Sección Antropología, 13(87), 125-136.

Kaufmann, C. A., Bellinzoni, J, Álvarez, M. C., González, M. E., Favier Dubois, C. y Barros, M. P. (2019). Aportes del estudio de los procesos de formación de sitio para el análisis de los restos humanos del sitio Laguna Muscar 2 (Lamadrid, Buenos Aires). Comechingonia Revista de Arqueología, 23(1), 325-348.

Kelt, D. A. (2011). Comparative ecology of desert small mammals: a selective review of the past 30 years. Journal of Mammalogy, 92, 1158-1178.

Kibii, J. M. (2009). Taphonomic aspects of African porcupines (Hystrix cristata) in the Kenyan Highlands. Journal of Taphonomy, 7, 21-27

Klippel. W. E. y Synstelien, J. A. (2007). Rodents as taphonomic agents: Bone gnawing by brown rat sand gray squirrels. Journal of Forensic Sciences, 52, 765773.

Lanzelotti, S. L. y Bonaparte, J. F. (2009). Contexto 
geoestratigráfico y procesos de formación del registro arqueológico en Cañada Honda: apuntes para su discusión y abordaje. En E. E. Fucks, C. Deschamps, C. G. Silva y E. J. Schnack (Eds.), IV Congreso Argentino de Cuaternario y Geomorfología, XII Congresso da Associação Brasileira de Estudos do Quaternário, II Reunión sobre el Cuaternario de América del Sur (pp. 247-258). La Plata.

Llanos, A. C. y Crespo, J. A. (1952). Ecología de la vizcacha (Lagostomus maximus maximus Blainv.) en el nordeste de la Provincia de Entre Ríos. Revista de Investigaciones Agrícolas, 6, 289-379.

Lyman, R. L. (1994). Vertebrate Taphonomy. Cambridge: Cambridge University Press.

Lyman, R. L. (2008). Quantitative Paleozoology. Cambridge: Cambridge University Press.

Madden, R. H. (2015). Hypsodonty in Mammals: Evolution, Geomoprhology, and the Role of Earth Surface Processes. Cambridge: Cambridge University Press.

Maguire, J. M. (1976). A Taxonomic and Ecological Study of the Living and Fossil Hystriceda with Particular reference to Southern Africa. Tesis Doctoral Inédita. Johannesburgo: University of Witwatersrand.

Maguire, J. M., Pemberton, D. y Collett, M. H. (1980). The Makapansgat Limeworks grey breccia: hominids, hyaenas, hystricids or hillwash, Palaeontologia Africana, 23, 75-98.

Mares, M. A. y Ojeda, R. A. (1984). Faunal commercialization and conservation in South America. Bioscience, 34, 580584.

Martínez, G. A. y Gutiérrez, M. A. (2004). Tendencias en la explotación humana de la fauna durante el Pleistoceno Final-Holoceno en la región pampeana (Argentina). En G. L. Mengoni Goñalons (Ed.), Zooarchaeology of South America (pp.81-98). Oxford: British Archaeological Research International Series.

Martínez, G. A., Messineo, P. G., Piñeyro, M. E., Kaufmann, C. A. y Barros, M. P. (2001). Análisis preliminar de la estructura faunística del sitio Paso Otero 3 (Pdo. de Necochea, Pcia. de Bs. As., Argentina). En Arqueología Uruguaya hacia el fin del milenio. IX Congreso Nacional De Arqueología (pp. 505-520). Montevideo: Asociación Uruguaya de Arqueología y Fundación Fontaina Minelli.

Massigoge, A., Gutiérrez, M. A., Alvarez, M. C., Kaufmann, C. A., Rafuse, D. J. y González, M. E. (2014). Estudio comparativo de las marcas de dientes producidas por dos pequeños carnívoros sudamericanos. Revista
Chilena de Antropología, 30, 42-49.

Massigoge, A., Rodríguez, M. N., Rafuse, D. J., Torino, R. F., Favier Dubois, C. y Steffan, P. G. (2018). Investigaciones arqueológicas en el sitio Las Brusquillas 3 (Holoceno tardío, región Pampeana, Argentina). Arqueología, 24(1), 147-171.

Mazzanti, D. y Quintana, C. (2001). Cueva Tixi: Cazadores y Recolectores de las Sierras de Tandilia Oriental. Geología, Paleontología y Zooarqueología. Mar del Plata: Laboratorio de Arqueología, Universidad Nacional de Mar del Plata, Publicación Especial 1.

Mengoni Goñalons, G. (1999). Cazadores de Guanaco de la Estepa Patagónica. Buenos Aires: Sociedad Argentina de Antropología.

Messineo, P. G., Álvarez, M. C., Favier Dubois, C., Steffan, P. y Colantonio, M. J. (2013). Estado de avance de las investigaciones arqueológicas en el sitio Empalme Querandíes 1 (centro de la subregión Pampa Húmeda, provincia de Buenos Aires). Comechingonia Revista de Arqueología, 17(1), 123-148.

Oshima, H., Nakasone, N., Hashimoto, E., Sakai, H., Nakakura-Ohshima, K. y Harada, H. (2005). The eternal tooth germ is formed at the apical end of continuously growing teeth. Archives of Oral Biology, 50(2), 153157.

Pardiñas, U. F. J. (1999). Tafonomía de Microvertebrados en Yacimientos Arqueológicos de Patagonia (Argentina). Revista Arqueología, 9, 265-340.

Platt, B. F., Kolb, D. J., Kunhardt, C. G., Milo, S. P. y New. L. G. (2016). Burrowing Through the Literature: The Impact of Soil-Disturbing Vertebrates on Physical and Chemical Properties of Soil. Soil Science, 181, 175-191.

Pocheville A. (2015). The ecological niche: History and recent controversies. En Th- Heams, P. Huneman, G. Lecointre y M. Silberstein (Eds.), Handbook of Evolutionary Thinking in the Sciences (pp. 547-586). Sidney: Springer Science.

Politis, G. G. (2014). Introducción. En G. G. Politis, M. A. Gutiérrez y C. Scabuzzo (Eds.), Estado Actual de Las Investigaciones en El Sitio Arqueológico Arroyo Seco 2 (Partido de Tres Arroyos, Provincia de Buenos Aires, Argentina) (pp. 11-28). Olavarría: Universidad Nacional del Centro de la Provincia de Buenos Aires.

Politis, G. G. y Madrid, P. (1988). Un hueso duro de roer: análisis preliminar de la tafonomía del sitio Laguna Tres Reyes 1, Pdo. de Adolfo Gonzáles Chaves, Pcia. de Buenos Aires. En A. Haber y N. Ratto (Eds.), De Procesos, Contextos y otros Huesos (pp. 29-44). Buenos Aires: 
Instituto de Ciencias Antropológicas, Universidad de Buenos Aires.

Politis, G. G. y Messineo, P. G. (2008). The Campo Laborde site: new evidence for the Holocene survival of Pleistocene megafauna in the Argentine Pampas. Quaternary International, 191, 98-114.

Quintana, C. (2005). Despiece de micro-roedores en el Holoceno Tardío de las Sierras de Tandilia (Argentina). Archaeofauna, International Journal of Archaeozoology 14, 227-241.

Quintana, C. (2007). Marcas de dientes de roedores en huesos de sitios arqueológicos de las sierras de Tandilia, Argentina. Archaeofauna, International Journal of Archaeozoology 16, 185-191.

Quintana, C. y Mazzanti, D. (2011). Las vizcachas pampeanas (Lagostomus maximus, Rodentia) en la subsistencia indígena del Holoceno tardío de las Sierras de Tandilia Oriental (Argentina). Latin American Antiquity, 22(2), 253-270.

Rafuse, D. J., González, M. E., Kaufmann, C. A., Álvarez, M. C., Gutiérrez, M. A. y Massigoge, A. (2014). Análisis comparativo de los patrones de modificaciones óseas de dos carnívoros sudamericanos: el gato montés (Leopardus geoffroyi) y el zorro pampeano (Lycalopex gymnocercus). Aportes para la identificación de la acción de pequeños carnívoros en el registro arqueológico. Magallania, 4 (1), 167-186.

Rafuse, D., Kaufmann, C. A., Gutiérrez, M. A., González, M., Scheifler, N., Álvarez, M. C. y Massigoge, A. (2019). Taphonomy of modern communal burrow systems of the Plains vizcacha (Lagostomus maximus, Chinchillidae) in the Pampas region of Argentina: implications for the fossil record. Historical Biology, 31(5), 517-534.

Salemme, M. y Madrid, P. (2007). The archaeofaunas from Laguna Tres Reyes 1 site: taxonomic richness and abundance during the beginning of the Late Holocene in the south-east Pampean Region (Argentina). En M. Gutiérrez, L. Miotti, G. Barrientos, G. L. Mengoni Goñalons y M. Salemme (Eds.), Taphonomy and Zooarchaeology in Argentina (pp. 121-143). Oxford: BAR International Series 1601

Salemme, M., Escosteguy, P. y Frontini, R. (2012). La fauna menor en sitios arqueológicos de la región pampeana, Argentina. Agente disturbador vs. recurso económico. Archaeofauna, International Journal of Archaeozoology 21(3), 163-185.

Scheifler, N., Álvarez, M. C., Rafuse, D., Kaufmann, C. A., Massigoge, A., González, M. y Gutiérrrez, M. A. (2020). Taphonomic signature of Geoffroy's cat (Leopardus geoffroyi) on small sized preys: A comparative study of ingested and non-ingested leporid bones. Journal of Archaeological Science: Reports, 31, 102340.

Tapaltsyan, V., Eronen, J. T., Lawing, M., Sharir, A., Janis, C., Jernvall, J. y Klein, O. D. (2015). Continuously Growing Rodent Molars Result from a Predictable Quantitative Evolutionary Change over 50 Million Years. Cell Reports, 11, 673-680.

Thornton, M. y Fee, J. (2001). Rodent gnawing as a taphonomic agent: Implications for archaeology. En S. C. Gerlach y M. S. Murray (Eds.), People and Wildlife in Northern America: Essays in Honor of $R$. Dale Guthrie (pp. 300-306). Oxford: BAR International Series 944.

Todd, L. C. (1987). Taphonomy of The Horner II bone bed. En G. C. Frison y L. C. Todd (Eds.), The Horner Site: The Type Site of the Cody Cultural Complex (107-198). Orlando: Academy Press.

Todd, L. C. y Frison, G. (1992). Reassembly of bison skeleton from the Horner Site: A study of anatomical refitting. En J. Hofman y J. Enloe (Eds.), Piecing together the Past: Applications of Refitting Studies in Archaeology (pp. 63-82). Oxford: BAR International Series 578.

Todd, L. C. y Stanford, D. (1992). Application of conjoined bone data to site structural studies. En J. Hofman y J. Enloe (Eds.), Piecing together the Past: Applications of Refitting Studies in Archaeology (21-35). Oxford: BAR International Series 578.

Tomassini, R. L., Montalvo, C., Fernández-Jalvo, Y., Garrone, M. C. y Kin, M. S. (2019). Modern plains vizcacha (Lagostomus maximus, Chinchillidae, Rodentia) as a bone accumulating agent in the Argentine Pampas: Application to the study of fossiliferous sites. Journal of Arid Environments, 161, 11-24.

Tong, H. W., Zhang, S., Chen, F. y Q. Li. (2008). Rongements sélectifs des os par les porcs-épics et autres rongeurs: cas de la grotte Tianyuan, un site avec des restes humains fossiles récemment découvert près de Zhoukoudian (Choukoutien). L'anthropologie, 112, 353-369.

Villarreal, D., Clark, K. L., Branch, L. C., Hierro, J. L. y Machicote, M. (2008). Alteration of ecosystem structure by a burrowing herbivore, the plains vizcacha (Lagostomus maximus). Journal of Mammalogy, 89, 700-711.

Waters, M. R. (1992). Principles of Geoarchaeology. A North American Perspective. Arizona: The University of Arizona Press.

Weir, B. J. (1974). Reproductive characteristics of 
hystricomorph rodents. Symposia of the Zoological Society of London, 34, 265-301.

Wood, W. R. y Johnson, D.L. (1978). A survey of disturbance processes in archaeological site formation. Advances in Archaeological Method and Theory, 1, 315-
381.

Zárate, M., Kemp, R. A., Espinosa, M. y Ferrero, L. (2000). Pedosedimentary and palaeoenvironmental significance of a Holocene alluvial sequence in the southern Pampas, Argentina. The Holocene, 10(4), 481-488. 